\title{
Asymmetric Perfectly Matched Layer for the Absorption of Waves ${ }^{1}$
}

\author{
Jean-Luc Vay* \\ * Accelerator and Fusion Research Division, Lawrence Berkeley National \\ Laboratory, Berkeley, California 94720 \\ E-mail: jlvay@lbl.gov
}

Version: July 22, 2002

The Perfectly Matched Layer (PML) has become a standard for comparison in the techniques that have been developed to close the system of Maxwell equations (more generally wave equations) when simulating an open system. The original Berenger PML formulation relies on a split version of Maxwell equations with numerical electric and magnetic conductivities. We present here an extension of this formulation which introduces counterparts of the electric and magnetic conductivities affecting the term which is spatially differentiated in the equations. The phase velocity along each direction is also multiplied by an additional coefficient. We show that, under certain constraints on the additional numerical coefficients, this "medium" does not generate any reflection at any angle and any frequency and is then a Perfectly Matched Layer. Technically it is a superset of Berenger's PML to which it reduces for a specific set of parameters and like it, it is anisotropic. However, unlike the PML, it introduces some asymmetry in the absorption rate and is therefore labeled an APML for Asymmetric Perfectly Matched Layer. We present here the numerical considerations that have led us to introduce such a medium as well as its theory. Several finite-difference numerical implementations are derived (in one, two and three dimensions) and the performance of the APML is contrasted with that of the PML in one and two dimensions. Using plane wave analysis, we show that our APML implementations lead to higher absorption rates than the considered PML implementations. Although we have considered in this paper the finite-difference discretization of Maxwell-like equations only, the APML system of equations may be used with other discretization schemes, such as finite-elements, and may be applied to other equations, for applications beyond electromagnetics.

Key Words: perfectly matched layer; PML; absorbing boundary condition; ABC; Finite-Difference Time-Domain; FDTD; wave equation; electromagnetics; Maxwell

\section{INTRODUCTION}

Often, part or all of a simulated region is modeled as if extended to infinity and an open boundary condition must be applied to close the system. In many cases, the open boundary condition consists of absorbing everything that comes out of the simulated region with, ideally, no reflection, and is then called an Absorbing Boundary Condition. For the wave equation, most ABCs can be grouped into two categories: "One-Way" ABCs that factorize the wave equation and extract an

\footnotetext{
${ }^{1}$ Supported by the Office of Fusion Energy, U.S. Department of Energy, under Contract DEACO3,76SF00098
} 
operator that permits only outgoing waves (higher order scheme can be derived by using products of one-way operators) or $\mathrm{ABCs}$ that use an absorbing layer which damps the outgoing waves. High order algorithms using the absorbing layer technique have been first obtained by Berenger using a split formulation of the wave equation, known as PML for Perfectly Matched Layer[2]. A lot of work has since been published concerning the PML technique and the reader can refer to $[1,3,6,7,9,13]$, just to cite a few. Good reviews of ABCs are given in $[8,6,7]$.

In [10], we have presented a formulation of a centered finite-differenced form of the wave equation which can be tuned to describe either a one-way operator or a PML layer. Moreover, we have presented a new ABC using this formulation, which presents characteristics of both one-way and PML ABCs. We have shown that the hybridization was beneficial. In this article, we generalize this approach and present a new kind of PML which is asymmetric, and is then labeled APML for Asymmetric Perfectly Matched Layer. By construction, it is a superset of Berenger's PML, to which it reduces for a specific choice of free parameters. Ignoring discretization errors the APML, like the PML, does not generate any reflection at any frequency and any angle following a specific prescription of parameters. Unlike the PML, it generally damps the wave asymmetrically. This asymmetrization is due to an additional numerical term relative to the Berenger split-field formulation. This term is formally symmetric to the damping term of the PML but acts on the quantity which is differentiated in space rather than the one that acts in time. As already noted in [11], this term appears naturally when recovering the infinitesimal limit of a centered finite-differenced formulation of the wave equation, which can be established using a minimal set of geometrical and operational requirements. An additional parameter, which is fixed in the PML formulation, but is free in the APML one is the wave speed for each direction of propagation.

At the infinitesimal limit, the coefficient of reflection of an APML layer is the same as that of a PML layer, provided a scaling of the conductivities, and is independent of the third free parameter which has been introduced into the equations. At this point, the introduction of the additional parameters seems pointless for the absorption of outgoing waves. However, the finite-difference implementation of the APML does not behave as its infinitesimal counterpart does. We present several possible implementations of the APML in finite-difference (including the one presented in [10]) and analyse their response to the excitation of a plane wave at an incoming angle by means of a coefficient of reflection, depending on the frequency and angle of the incident wave. The results are contrasted with a classic implementation of the PML as given in [2] and an implementation of the PML of our own that we presented in [10]. They show that, for plane waves, our APML implementations provide higher absorption rates than the considered PML implementations.

\subsection{Notations}

In this article, we will consider quantities discretized on orthogonal and regular space-time grids. On such a grid, a quantity $A$ will be denoted $A_{j}^{i}, A_{j k}^{i}$ or $A_{j k l}^{i}$ for respectively, a 1-, 2- or 3-dimensional system, where $\mathrm{i}$ is the time index and $\mathrm{j}, \mathrm{k}$ and $\mathrm{l}$ are the space indices along $\mathrm{x}, \mathrm{y}$ and $\mathrm{z}$. Note that a location defined half way between $j$ and $j+1$ will be noted $j+1 / 2$. On such a grid, we define the operators of centered finite difference $\Delta_{u}$ and finite average $\Sigma_{u}$ 


$$
\begin{aligned}
\Delta_{t} A & =\frac{A_{j, k, l}^{i+1 / 2}-A_{j, k, l}^{i-1 / 2}}{\delta t} \\
\Delta_{x} A & =\frac{A_{j+1 / 2, k, l}^{i}-A_{j-1 / 2, k, l}^{i}}{\delta t} \\
\Sigma_{t} A & =\frac{A_{j, k, l}^{i+1 / 2}+A_{j, k, l}^{i-1 / 2}}{2} \\
\Sigma_{x} A & =\frac{A_{j+1 / 2, k, l}^{i}+A_{j-1 / 2, k, l}^{i}}{2}
\end{aligned}
$$

where $u=(x, y$ or $z), \delta t$ is the time step and $\delta_{u}$ is the mesh size along $u$.

\subsection{General considerations on explicit discrete representation of the wave equation}

We give some general considerations on explicit calculation of the wave equation on a discrete space-time grid that have led us to the introduction of the APML medium (these considerations were already given in [11]).

For simplicity, we restrict this part to the study of a one dimensional waveequation in vacuum of the form

$$
\frac{\partial^{2} E}{\partial t^{2}}=c^{2} \frac{\partial^{2} E}{\partial x^{2}}
$$

which is equivalent to the system

$$
\begin{aligned}
& \frac{\partial E}{\partial t}=c \frac{\partial B}{\partial x} \\
& \frac{\partial B}{\partial t}=c \frac{\partial E}{\partial x}
\end{aligned}
$$

We consider solving this system in a discrete space and use the Yee discretization scheme [12] where $E$ and $B$ are staggered in space as well as in time (see Fig. 1). If we assume that the discrete solution is explicit (i.e. the evaluation of a quantity at a given time step involves only quantities known at previous time steps) and is linear, we then have

$$
\begin{aligned}
& \begin{array}{rlllll}
E_{j}^{i+1} & =\alpha_{1} E_{j}^{i} & +\beta_{11} B_{j+1 / 2}^{i+1 / 2} & -\beta_{12} B_{j-1 / 2}^{i+1 / 2} & \ldots+\beta_{1 n} B_{j+n / 2}^{i+1 / 2} & \ldots \\
& +\alpha_{2} E_{j}^{i-1} & +\beta_{21} B_{j+1 / 2}^{i-1 / 2} & -\beta_{22} B_{j-1 / 2}^{i-1 / 2} & \ldots+\beta_{2 n} B_{j+n / 2}^{i-1 / 2} & \ldots
\end{array} \\
& \begin{array}{llllll}
\cdots & \cdots & \cdots & \cdots & & \cdots \\
+\alpha_{m} E_{j}^{i-m+1} & +\beta_{m 1} B_{j+1 / 2}^{i-m+3 / 2} & -\beta_{m 2} B_{j-1 / 2}^{i-m+3 / 2} & \cdots+\beta_{m n} B_{j+n / 2}^{i-m+3 / 2} & \cdots
\end{array} \\
& \begin{array}{lllll} 
& \ldots & \ldots & \ldots & \ldots
\end{array}
\end{aligned}
$$

where $\alpha_{m}$ and $\beta_{m n}$ are constants.

If we add the constraint that the equation should be minimal and symmetric, then the most compact possibility is given by

$$
E_{j}^{i+1}=\alpha E_{j}^{i}+\beta_{p} B_{j+1 / 2}^{i+1 / 2}-\beta_{m} B_{j-1 / 2}^{i+1 / 2}
$$


where $\alpha, \beta_{p}$ and $\beta_{m}$ are three constants. For the modeling of (5), we present two ways to determine these constants.

One way is to consider a wave $e^{i(\omega t-k x)}$ and use it in (9)

$$
e^{i \omega \delta t / 2}=\alpha e^{-i \omega \delta t / 2} \pm \beta_{p} e^{-i k \delta x / 2} \mp \beta_{m} e^{i k \delta x / 2}
$$

where the \pm and $\mp$ discriminate the cases of waves propagating forward or backward.

We then make a long wavelength approximation and assume that $\omega \delta t \ll 1$ and $k \delta x \ll 1$, so that we can expand and truncate the exponentials, giving

$$
1+i \omega \delta t / 2=\alpha(1-i \omega \delta t / 2) \pm \beta_{p}(1-i k \delta x / 2) \mp \beta_{m}(1+i k \delta x / 2)
$$

Requiring $\omega$ and $k$ to be real (propagation in vacuum) and $\omega=k c$, we can separate the real and imaginary parts, yielding a system of four equations that, once solved, gives

$\alpha=1$ and $\beta_{p}=\beta_{m}=c \delta t / \delta x$.

Another way relies on the fact that (9) can be rewritten

$$
\frac{E_{j}^{i+1}-E_{j}^{i}}{\delta t}+\sigma_{E} \frac{E_{j}^{i+1}+E_{j}^{i}}{2}=c_{E} \frac{B_{j+1 / 2}^{i+1 / 2}-B_{j-1 / 2}^{i+1 / 2}}{\delta x}+\sigma_{B} \frac{B_{j+1 / 2}^{i+1 / 2}+B_{j-1 / 2}^{i+1 / 2}}{2}
$$

with

$$
\left\{\begin{array}{l}
\sigma_{E}=\frac{2}{\delta t} \frac{1-\alpha}{1+\alpha} \\
c_{E}=\frac{\delta x}{\delta t} \frac{\beta_{p}+\beta_{m}}{1+\alpha} \\
\sigma_{B}=\frac{2}{\delta t} \frac{\beta_{p}-\beta_{m}}{1+\alpha}
\end{array}\right.
$$

At the infinitesimal limit, (11) becomes

$$
\frac{\partial E}{\partial t}+\sigma_{E} E=c_{E} \frac{\partial B}{\partial x}+\sigma_{B} B
$$

which will describe a wave equation in vacuum only if $\sigma_{E}=\sigma_{B}=0$ and $c_{E}=c$, giving $\alpha=1$ and $\beta_{p}=\beta_{m}=c \delta t / \delta x$.

The term $\sigma_{E}$ has a physical meaning, but that is not the case for $\sigma_{B}$ which may appear as an undesirable term. It turns out that, as we have shown in [10] and [11], this term plays a role in the discretized form of (5). For example, the Sommerfeld outgoing-wave boundary condition is given by (for waves propagating forward)

$$
E_{j}^{i+1}=\left(1-\frac{2 c \delta t}{c \delta t+\delta x}\right) E_{j}^{i}-\frac{2 c \delta t}{c \delta t+\delta x} B_{j-1 / 2}^{i+1 / 2}
$$

which rewrites

$\frac{E_{j}^{i+1}-E_{j}^{i}}{\delta t}+\frac{2 c}{\delta x} \frac{E_{j}^{i+1}+E_{j}^{i}}{2}=c \frac{B_{j+1 / 2}^{i+1 / 2}-B_{j-1 / 2}^{i+1 / 2}}{\delta x}-\frac{2 c}{\delta x} \frac{B_{j+1 / 2}^{i+1 / 2}+B_{j-1 / 2}^{i+1 / 2}}{2}$

and has the same form as (11). 


\section{THEORY OF THE ASYMMETRIC PERFECTLY MATCHED LAYER}

In order to understand the implications of the additional coefficient $\sigma_{B}$ in (13), we have introduced it as an additional term in a split form of Maxwell equations, following Berenger's presentation of the PML medium [2]. As the analysis will reveal, the resulting medium has properties similar to a PML medium (to which it reduces for a choice of parameters) although it introduces some asymmetry in the coefficient of absorption.

\subsection{Definition of the APML medium}

For the TE case, we define the APML as

$$
\begin{aligned}
\varepsilon_{0} \frac{\partial E_{x}}{\partial t}+\sigma_{y} E_{x} & =\frac{c_{y}}{c} \frac{\partial H_{z}}{\partial y}+\bar{\sigma}_{y} H_{z} \\
\varepsilon_{0} \frac{\partial E_{y}}{\partial t}+\sigma_{x} E_{y} & =-\frac{c_{x}}{c} \frac{\partial H_{z}}{\partial x}+\bar{\sigma}_{x} H_{z} \\
\mu_{0} \frac{\partial H_{z x}}{\partial t}+\sigma_{x}^{*} H_{z x} & =-\frac{c_{x}^{*}}{c} \frac{\partial E_{y}}{\partial x}+\bar{\sigma}_{x}^{*} E_{y} \\
\mu_{0} \frac{\partial H_{z y}}{\partial t}+\sigma_{y}^{*} H_{z y} & =\frac{c_{y}^{*}}{c} \frac{\partial E_{x}}{\partial y}+\bar{\sigma}_{y}^{*} E_{x} \\
H_{z} & =H_{z x}+H_{z y}
\end{aligned}
$$

For $c_{x}=c_{y}=c_{x}^{*}=c_{y}^{*}=c$ and $\bar{\sigma}_{x}=\bar{\sigma}_{y}=\bar{\sigma}_{x}^{*}=\bar{\sigma}_{y}^{*}=0$, this system reduces to the Berenger PML medium, while adding the additional constraint $\sigma_{x}=\sigma_{y}=$ $\sigma_{x}^{*}=\sigma_{y}^{*}=0$ leads to the system of Maxwell equations in vacuum.

\subsection{Propagation of a Plane Wave in an APML Medium}

We consider a plane wave of magnitude $\left(E_{0}, H_{z x 0}, H_{z y 0}\right)$ and pulsation $\omega$ propagating in the APML medium with an angle $\varphi$ relative to the $\mathrm{x}$ axis

$$
\begin{aligned}
E_{x} & =-E_{0} \sin \varphi e^{i \omega(t-\alpha x-\beta y)} \\
E_{y} & =E_{0} \cos \varphi e^{i \omega(t-\alpha x-\beta y)} \\
H_{z x} & =H_{z x 0} e^{i \omega(t-\alpha x-\beta y)} \\
H_{z y} & =H_{z y 0} e^{i \omega(t-\alpha x-\beta y)}
\end{aligned}
$$

where $\alpha$ and $\beta$ are two complex constants to be determined.

Introducing (21), (22), (23) and (24) into (16), (17), (18) and (19) gives

$$
\begin{aligned}
\varepsilon_{0} E_{0} \sin \varphi-i \frac{\sigma_{y}}{\omega} E_{0} \sin \varphi & =\beta \frac{c_{y}}{c}\left(H_{z x 0}+H_{z y 0}\right)+i \frac{\bar{\sigma}_{y}}{\omega}\left(H_{z x 0}+H_{z y 0}\right) \\
\varepsilon_{0} E_{0} \cos \varphi-i \frac{\sigma_{x}}{\omega} E_{0} \cos \varphi & =\alpha \frac{c_{x}}{c}\left(H_{z x 0}+H_{z y 0}\right)-i \frac{\bar{\sigma}_{x}}{\omega}\left(H_{z x 0}+H_{z y 0}\right) \\
\mu_{0} H_{z x 0}-i \frac{\sigma_{x}^{*}}{\omega} H_{z x 0} & =\alpha \frac{c_{x}^{*}}{c} E_{0} \cos \varphi-i \frac{\bar{\sigma}_{x}^{*}}{\omega} E_{0} \cos \varphi \\
\mu_{0} H_{z y 0}-i \frac{\sigma_{y}^{*}}{\omega} H_{z y 0} & =\beta \frac{c_{y}^{*}}{c} E_{0} \sin \varphi+i \frac{\bar{\sigma}_{y}^{*}}{\omega} E_{0} \sin \varphi
\end{aligned}
$$


Defining $Z=E_{0} /\left(H_{z x 0}+H_{z y 0}\right)$ and using (25) and (26), we get

$$
\begin{aligned}
\beta & =\left[Z\left(\varepsilon_{0}-i \frac{\sigma_{y}}{\omega}\right) \sin \varphi-i \frac{\bar{\sigma}_{y}}{\omega}\right] \frac{c}{c_{y}} \\
\alpha & =\left[Z\left(\varepsilon_{0}-i \frac{\sigma_{x}}{\omega}\right) \cos \varphi+i \frac{\bar{\sigma}_{x}}{\omega}\right] \frac{c}{c_{x}}
\end{aligned}
$$

Adding $H_{z x 0}$ and $H_{z y 0}$ from (27) and (28) and substituting the expressions for $\alpha$ and $\beta$ from (29) and (30) yields

$$
\begin{aligned}
\frac{1}{Z} & =\frac{Z\left(\varepsilon_{0}-i \frac{\sigma_{x}}{\omega}\right) \cos \varphi \frac{c_{x}^{*}}{c_{x}}+i \frac{\bar{\sigma}_{x}}{\omega} \frac{c_{x}^{*}}{c_{x}}-i \frac{\bar{\sigma}_{x}^{*}}{\omega}}{\mu_{0}-i \frac{\sigma_{x}^{*}}{\omega}} \cos \varphi \\
& +\frac{Z\left(\varepsilon_{0}-i \frac{\sigma_{y}}{\omega}\right) \sin \varphi \frac{c_{y}^{*}}{c_{y}}-i \frac{\bar{\sigma}_{y}}{\omega} \frac{c_{y}^{*}}{c_{y}}+i \frac{\bar{\sigma}_{y}^{*}}{\omega}}{\mu_{0}-i \frac{\sigma_{y}^{*}}{\omega}} \sin \varphi
\end{aligned}
$$

If $c_{x}=c_{x}^{*}, c_{y}=c_{y}^{*}, \bar{\sigma}_{x}=\bar{\sigma}_{x}^{*}, \bar{\sigma}_{y}=\bar{\sigma}_{y}^{*}, \frac{\sigma_{x}}{\varepsilon_{0}}=\frac{\sigma_{x}^{*}}{\mu_{0}}$ and $\frac{\sigma_{y}}{\varepsilon_{0}}=\frac{\sigma_{y}^{*}}{\mu_{0}}$ then

$$
Z= \pm \sqrt{\frac{\mu_{0}}{\varepsilon_{0}}}
$$

which is the impedance of vacuum. Hence, like the PML, given some restrictions on the parameters, the APML does not generate any reflection at any angle and any frequency. As for the PML, this property is not retained after discretization, as shown subsequently in this paper.

Calling $\psi$ any component of the field and $\psi_{0}$ its magnitude, we get from (21), (29), (30) and (32) that

$$
\psi=\psi_{0} e^{i \omega\left(t \mp x \cos \varphi / c_{x} \mp y \sin \varphi / c_{y}\right)} e^{-\left( \pm \frac{\sigma_{x} \cos \varphi}{\varepsilon_{0} c_{x}}+\bar{\sigma}_{x} \frac{c}{c_{x}}\right) x} e^{-\left( \pm \frac{\sigma_{y} \sin \varphi}{\varepsilon_{0} c_{y}}+\bar{\sigma}_{y} \frac{c}{c_{y}}\right) y}
$$

We assume that we have an APML layer of thickness $\delta$ (measured along $x$ ) and that $\sigma_{y}=\bar{\sigma}_{y}=0$ and $c_{y}=c$. Using (33), we determine that the coefficient of reflection given by this layer is

$$
\begin{aligned}
R_{A P M L}(\theta) & =e^{-\left(\sigma_{x} \cos \varphi / \varepsilon_{0} c_{x}+\bar{\sigma}_{x} c / c_{x}\right) \delta} e^{-\left(\sigma_{x} \cos \varphi / \varepsilon_{0} c_{x}-\bar{\sigma}_{x} c / c_{x}\right) \delta} \\
& =e^{-2\left(\sigma_{x} \cos \varphi / \varepsilon_{0} c_{x}\right) \delta}
\end{aligned}
$$

which happens to be the same as the PML theoretical coefficient of reflection if we assume $c_{x}=c$. Hence, it follows that for the purpose of wave absorption, the term $\bar{\sigma}_{x}$ seems to be of no interest. However, although this conclusion is true at the infinitesimal limit, it does not hold for the discretized counterpart.

\section{DISCRETIZATION OF THE APML}

In this section, we derive several possible finite-difference discretizations of (16) to (19) in one dimension, followed by the extension to higher dimension. The reader who is not interested in the details of these derivations may jump to the next section and find a concised list of the proposed discretized schemes into Appendix B. 


\subsection{In one dimension}

Equations (16), (17), (18) and (19) have all the form of a one-dimensional equation

$$
\frac{\partial F}{\partial t}+\sigma_{u} F=c_{u} \frac{\partial G}{\partial u}+\bar{\sigma}_{u} G
$$

\subsubsection{Exponential time and space stepping}

A possible implementation consists of applying the exponential time-stepping method [6] to time and space. The discretization of

$$
\frac{\partial F}{\partial t}+\sigma_{u} F
$$

using the exponential time-stepping method is given by

$$
\sigma_{u} \frac{F_{j}^{i+1}-e^{-\sigma_{u} \delta t} F_{j}^{i}}{1-e^{-\sigma_{u} \delta t}}
$$

Similarly, we can apply the method in space rather than in time and the discretization of $c_{u} \frac{\partial G}{\partial u}+\bar{\sigma}_{u} G$ is then given by

$$
\frac{\bar{\sigma}_{u}}{c_{u}} \frac{G_{j+1 / 2}^{i+1 / 2}-e^{-\bar{\sigma}_{u} \delta u / c_{u}} G_{j-1 / 2}^{i+1 / 2}}{1-e^{-\bar{\sigma}_{u} \delta u / c_{u}}}
$$

Merging the two expressions gives

$$
F_{j}^{i+1}=e^{-\sigma_{u} \delta t} F_{j}^{i}+\frac{\bar{\sigma}_{u}}{\sigma_{u}} \frac{1-e^{-\sigma_{u} \delta t}}{1-e^{-\bar{\sigma}_{u} \delta u / c_{u}}}\left(G_{j+1 / 2}^{i+1 / 2}-e^{-\bar{\sigma}_{u} \delta u / c_{u}} G_{j-1 / 2}^{i+1 / 2}\right)
$$

which is of the form

$$
F_{j}^{i+1}=\alpha F_{j}^{i}+\beta_{p} G_{j+1 / 2}^{i+1 / 2}-\beta_{m} G_{j-1 / 2}^{i+1 / 2}
$$

with

$$
\begin{aligned}
\alpha & =e^{-\sigma_{u} \delta t} \\
\beta_{p} & =\frac{\bar{\sigma}_{u}}{\sigma_{u}} \frac{1-e^{-\sigma_{u} \delta t}}{1-e^{-\bar{\sigma}_{u} \delta u / c_{u}}} \\
\beta_{m} & =e^{-\bar{\sigma}_{u} \delta u / c_{u}} \beta_{p}
\end{aligned}
$$

In the form of (11), (40) becomes

$\frac{F_{j}^{i+1 / 2}-F_{j}^{i-1 / 2}}{\delta t}+\sigma_{u}^{d} \frac{F_{j}^{i+1 / 2}+F_{j}^{i-1 / 2}}{2}=c_{u}^{d} \frac{G_{j+1 / 2}^{i}-G_{j-1 / 2}^{i}}{\delta u}+\bar{\sigma}_{u}^{d} \frac{G_{j+1 / 2}^{i}+G_{j-1 / 2}^{i}}{2}$

where 


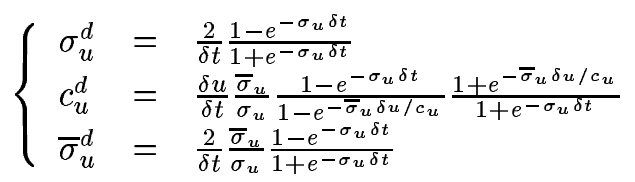

For consistency, we can verify that at the infinitesimal limit, $\sigma_{u}^{d} \rightarrow \sigma_{u}, c_{u}^{d} \rightarrow c_{u}$ and $\bar{\sigma}_{u}^{d} \rightarrow \bar{\sigma}_{u}$. This implementation will be labeled APML-Exponential.

\subsubsection{Direct assignment of coefficients}

We present here a different implementation. Instead of directly deriving a discrete approximation of the infinitesimal equation being modeled, we pose the form of the algorithm and assign the coefficients so that the resulting algorithm matches properties of the equation it is modeling.

Following the considerations of 1.2 , we pose the form to be

$$
F_{j}^{i+1 / 2}=\alpha F_{j}^{i-1 / 2}+\beta_{p} G_{j+1 / 2}^{i}-\beta_{m} G_{j-1 / 2}^{i}
$$

We have seen in 2.2 that in an APML medium, a wave experiences a modification of its amplitude as it propagates and that the amplitude of the modification is dependent on the direction of propagation. Let us define $t_{j}^{+}$and $t_{j}^{-}$to be the coefficients of transmission at location $j$ for waves propagating respectively forward and backward. Hence, a wave propagating forward having amplitude $A$ at location $j-1 / 2$ will have the amplitude $A t_{j}^{+}$at location $j$ and $A t_{j}^{+} t_{j+1 / 2}^{+}$at location $j+1 / 2$ while a wave propagating backward having amplitude $A$ at location $j+1 / 2$ will have the amplitude $A t_{j}^{-}$at location $j$ and $A t_{j}^{-} t_{j-1 / 2}^{-}$at location $j-1 / 2$.

We consider now a forward-propagative wave of the form $e^{i(\omega t-k x)}$, with amplitude $A$ at location $j-1 / 2$. At location $j$, we have from (46)

$$
A t_{j}^{+} e^{i \omega \delta t / 2}=\alpha A t_{j}^{+} e^{-i \omega \delta t / 2}-\beta_{p} A t_{j}^{+} t_{j+1 / 2}^{+} e^{-i k_{j+1 / 2}^{+} \delta x / 2}+\beta_{m} A e^{i k_{j-1 / 2}^{+} \delta x / 2}
$$

while considering a wave propagating backward gives

$$
A t_{j}^{-} e^{i \omega \delta t / 2}=\alpha A t_{j}^{-} e^{-i \omega \delta t / 2}+\beta_{p} A e^{-i k_{j+1 / 2}^{-} \delta x / 2}-\beta_{m} A t_{j}^{-} t_{j-1 / 2}^{-} e^{i k_{j-1 / 2}^{-} \delta x / 2}
$$

Steady state approximation Assuming a steady state approximation, the exponentials become unity and we get

$$
\alpha=1+\beta_{p} t_{j+1 / 2}^{+}-\beta_{m} / t_{j}^{+}
$$

for waves traveling forward and

$$
\alpha=1+\beta_{m} t_{j-1 / 2}^{-}-\beta_{p} / t_{j}^{-}
$$

for waves traveling backward.

Using, from (12)

$$
c_{u}^{d}=\frac{\delta u}{\delta t} \frac{\beta_{p}+\beta_{m}}{1+\alpha}
$$


and setting $c_{u}^{d}=c_{u}$, we can solve (49), (50) and (51) to get $\alpha, \beta_{p}$ and $\beta_{m}$

$$
\begin{aligned}
\alpha & =\frac{-1+\frac{\delta u}{c_{u} \delta t}\left(t_{p}+t_{m}+t_{p} t_{m}\left(t_{p p}+t_{m m}\right)\right)+t_{p} t_{m m} t_{p p} t_{m}}{1+\frac{\delta u}{c_{u} \delta t}\left(t_{p}+t_{m}+t_{p} t_{m}\left(t_{p p}+t_{m m}\right)\right)-t_{p} t_{m m} t_{p p} t_{m}} \\
\beta_{p} & =\frac{2 t_{m}\left(1+t_{m m} t_{p}\right)}{1+\frac{\delta u}{c_{u} \delta t}\left(t_{p}+t_{m}+t_{p} t_{m}\left(t_{p p}+t_{m m}\right)\right)-t_{p} t_{m m} t_{p p} t_{m}} \\
\beta_{m} & =\frac{2 t_{p}\left(1+t_{p p} t_{m}\right)}{1+\frac{\delta u}{c_{u} \delta t}\left(t_{p}+t_{m}+t_{p} t_{m}\left(t_{p p}+t_{m m}\right)\right)-t_{p} t_{m m} t_{p p} t_{m}}
\end{aligned}
$$

where we have used the notation

$$
\begin{aligned}
t_{p} & \equiv t_{j}^{+} \\
t_{m} & \equiv t_{j}^{-} \\
t_{p p} & \equiv t_{j+1 / 2}^{+} \\
t_{m m} & \equiv t_{j-1 / 2}^{-}
\end{aligned}
$$

for better readability.

Note that we also have

$$
\begin{aligned}
\sigma_{u}^{d} & =\frac{2 c_{u}}{\delta u} \frac{1-t_{p} t_{m m} t_{p p} t_{m}}{\left(t_{p}+t_{m}+t_{p} t_{m}\left(t_{p p}+t_{m m}\right)\right)} \\
c_{u}^{d} & =c_{u} \\
\bar{\sigma}_{u}^{d} & =\frac{2 c_{u}}{\delta u} \frac{t_{m}-t_{p}+t_{p} t_{m}\left(t_{m m}-t_{p p}\right)}{\left(t_{p}+t_{m}+t_{p} t_{m}\left(t_{p p}+t_{m m}\right)\right)}
\end{aligned}
$$

This implementation will be labeled APML-SSA for APML-Steady State Approximation.

Long-wavelength approximation Let us now make a long wavelength approximation of (47) and (48). We assume that $\omega \delta t \ll 1$ and $k \delta x \ll 1$ so that we can expand and truncate the exponentials and get

$$
\begin{aligned}
t_{j}^{+}(1+i \omega \delta t / 2) & =\alpha t_{j}^{+}(1-i \omega \delta t / 2) \\
& -\beta_{p} t_{j}^{+} t_{j+1 / 2}^{+}\left(1-i k_{j+1 / 2}^{+} \delta x / 2\right) \\
& +\beta_{m}\left(1+i k_{j-1 / 2}^{+} \delta x / 2\right)
\end{aligned}
$$

for waves propagating forward and

$$
\begin{aligned}
t_{j}^{-}(1+i \omega \delta t / 2) & =\alpha t_{j}^{-}(1-i \omega \delta t / 2) \\
& +\beta_{p}\left(1-i k_{j+1 / 2}^{-} \delta x / 2\right) \\
& -\beta_{m} t_{j}^{-} t_{j-1 / 2}^{-}\left(1+i k_{j-1 / 2}^{-} \delta x / 2\right)
\end{aligned}
$$


for waves propagating backward. We also impose the speed of waves to match the speed of the APML layer, that is that $k_{j}^{+}=k_{j}^{-}=k_{j}=\omega c_{j}$ for any $j$.

Since the coefficients of transmission take care of amplitude modifications, $\omega$ and $k$ are real, and separating the real and the imaginary parts of the two preceding equations, we get

$$
\begin{gathered}
\alpha=1+\beta_{p} t_{j+1 / 2}^{+}-\beta_{m} / t_{j}^{+} \\
\alpha=-1+\gamma_{j+1 / 2} \beta_{p} t_{j+1 / 2}^{+}+\gamma_{j-1 / 2} \beta_{m} / t_{j}^{+} \\
\alpha=1+\beta_{m} t_{j-1 / 2}^{-}-\beta_{p} / t_{j}^{-} \\
\alpha=-1+\gamma_{j-1 / 2} \beta_{m} t_{j-1 / 2}^{-}+\gamma_{j+1 / 2} \beta_{p} / t_{j}^{-}
\end{gathered}
$$

where $\gamma_{j+1 / 2}=\frac{\delta x}{c_{j+1 / 2} \delta t}$ and $\gamma_{j-1 / 2}=\frac{\delta x}{c_{j-1 / 2} \delta t}$.

We have obtained four independent equations linking $\alpha, \beta_{p}$ and $\beta_{m}$. We need to discard one if we do not want to impose any restrictions on the values of $t_{j}^{+}$, $t_{j}^{-}, t_{j+1 / 2}^{+}$and $t_{j-1 / 2}^{-}$. We want to retain (64) and (66) because they represent the lowest order of approximation. Assuming that we consider an APML layer for absorbing waves propagating forward, we are more interested in verifying the next order of approximation for waves propagating forward and will then keep (65) and disregard (67).

Then, we solve (64), (65) and (66) to obtain

$$
\begin{aligned}
\alpha & =\frac{-1+\gamma_{m}+t_{m} t_{p p}\left(\gamma_{p}+\gamma_{m}\right)+t_{p} t_{m m} t_{p p} t_{m}\left(\gamma_{p}+1\right)}{1+\gamma_{m}+t_{m} t_{p p}\left(\gamma_{p}+\gamma_{m}\right)+t_{p} t_{m m} t_{p p} t_{m}\left(\gamma_{p}-1\right)} \\
\beta_{p} & =\frac{2 t_{m}\left(1+t_{m m} t_{p}\right)}{1+\gamma_{m}+t_{m} t_{p p}\left(\gamma_{p}+\gamma_{m}\right)+t_{p} t_{m m} t_{p p} t_{m}\left(\gamma_{p}-1\right)} \\
\beta_{m} & =\frac{2 t_{p}\left(1+t_{p p} t_{m}\right)}{1+\gamma_{m}+t_{m} t_{p p}\left(\gamma_{p}+\gamma_{m}\right)+t_{p} t_{m m} t_{p p} t_{m}\left(\gamma_{p}-1\right)}
\end{aligned}
$$

where we have, as in the previous subsection, the same notation shortcut using $t_{p}, t_{m}, t_{p p}, t_{m m}$ as well as

$$
\begin{aligned}
\gamma_{m} & \equiv \gamma_{j-1 / 2} \\
\gamma_{p} & \equiv \gamma_{j+1 / 2}
\end{aligned}
$$

For completeness, we note that we also have

$$
\begin{aligned}
\sigma_{u}^{d} & =\frac{2}{\delta t} \frac{1-t_{p} t_{m m} t_{p p} t_{m}}{\gamma_{m}+t_{m} t_{p p}\left[\gamma_{m}+\gamma_{p}\left(1+t_{p} t_{m m}\right)\right]} \\
c_{u}^{d} & =\frac{\delta u}{\delta t} \frac{t_{m}+t_{p}+t_{p} t_{m}\left(t_{m m}+t_{p p}\right)}{\gamma_{m}+t_{m} t_{p p}\left[\gamma_{m}+\gamma_{p}\left(1+t_{p} t_{m m}\right)\right]} \\
\bar{\sigma}_{u}^{d} & =\frac{2}{\delta t} \frac{t_{m}-t_{p}+t_{p} t_{m}\left(t_{m m}-t_{p p}\right)}{\gamma_{m}+t_{m} t_{p p}\left[\gamma_{m}+\gamma_{p}\left(1+t_{p} t_{m m}\right)\right]}
\end{aligned}
$$


We now define $\sigma_{u, j}^{a}=\int_{-j-1 / 2}^{j} \sigma_{u} d u / \int_{-j-1 / 2}^{j} d u$ and $\bar{\sigma}_{u, j}^{a}=\int_{-j-1 / 2}^{j} \bar{\sigma}_{u} d u / \int_{-j-1 / 2}^{j} d u$. It follows that

$$
\begin{aligned}
t_{p} & =e^{-\left(\sigma_{u, j}^{a}+\bar{\sigma}_{u, j}^{a}\right) \delta u / 2} \\
t_{m m} & =e^{-\left(\sigma_{u, j}^{a}-\bar{\sigma}_{u, j}^{a}\right) \delta u / 2} \\
t_{p p} & =e^{-\left(\sigma_{u, j+1 / 2}^{a}+\bar{\sigma}_{u, j+1 / 2}^{a}\right) \delta u / 2} \\
t_{m} & =e^{-\left(\sigma_{u, j+1 / 2}^{a}-\bar{\sigma}_{u, j+1 / 2}^{a}\right) \delta u / 2}
\end{aligned}
$$

so that

$$
\begin{aligned}
t_{p} t_{m m} & =e^{-\sigma_{u, j}^{a} \delta u} \\
t_{p p} t_{m} & =e^{-\sigma_{u, j+1 / 2}^{a} \delta u}
\end{aligned}
$$

Rewriting (47) and (48) in terms of $t_{p}, t_{m}, t_{p p}$ and $t_{m m}$, gives

$$
\begin{aligned}
& e^{i \omega \delta t / 2}=\alpha e^{-i \omega \delta t / 2}-\beta_{p} t_{p p} e^{-i k_{p}^{+} \delta x / 2}+\beta_{m} A e^{i k_{m}^{+} \delta x / 2} / t_{p} \\
& e^{i \omega \delta t / 2}=\alpha e^{-i \omega \delta t / 2}+\beta_{p} e^{-i k_{p}^{-} \delta x / 2} / t_{m}-\beta_{m} t_{m m} e^{i k_{m}^{-} \delta x / 2}
\end{aligned}
$$

By substituting (68), (69), (70), (78) and (79) into (80) and (81), we remark that (80) and (81) are independent of $\bar{\sigma}_{u}^{a}$. As the numerical results will show, this property seem to hold in practical implementation. The wave reflected by an APML layer under this implementation seems to be independent of $\bar{\sigma}_{u}^{a}$, although the coefficients $\alpha, \beta_{p}$ and $\beta_{m}$ are different, which is quite a remarkable behavior. We note that it then reproduces a property of the APML which was demonstrated in 2.2 .

This implementation will be labeled APML-LWA for APML-Long Wavelength Approximation.

\subsubsection{Hybrid PML-Sommerfeld}

Another possibility relies on assigning the coefficients $\alpha, \beta_{p}$, and $\beta_{m}$ in (46) following the requirements that the algorithm must converge to:

- the standard Yee scheme $\left(\alpha=1, \beta_{p}=\beta_{m}=c \delta t / \delta x\right)$ when all the coefficients of transmission equal unity,

- the Sommerfeld outgoing-wave ABC $\left(\alpha=1-\beta_{m}, \beta_{p}=0, \beta_{m}=2 c \delta t /(\delta x+c \delta t)\right)$ when the coefficient of transmission of the next plane is zero.

An infinity of possibilities exist and we will only consider the one that we have presented in [10] which is given by (for a wave traveling in the forward direction)

$$
\begin{aligned}
\alpha & =1-\frac{c \delta t}{\delta x}\left[1+\left(\frac{\delta x-c \delta t}{\delta x+c \delta t}\right)\left(1-t_{j+1 / 2}^{+}\right)\right]+t_{j+1 / 2}^{+} \frac{c \delta t}{\delta x} \\
\beta_{p} & =\frac{c \delta t}{\delta x} \\
\beta_{m} & =t_{j}^{+} \frac{c \delta t}{\delta x}\left[1+\left(\frac{\delta x-c \delta t}{\delta x+c \delta t}\right)\left(1-t_{j+1 / 2}^{+}\right)\right]
\end{aligned}
$$

This implementation will be labeled APML-Hybrid. 


\subsection{Extension to 2 dimensions and beyond}

\subsubsection{The scalar wave equation}

We introduce the notation

$$
\begin{gathered}
\Gamma_{u}=\frac{\partial}{\partial t}-\sigma_{u} \\
\Lambda_{u}=c_{u} \frac{\partial}{\partial u}+\bar{\sigma}_{u}
\end{gathered}
$$

with $(u=x, y, z)$.

Assuming a three dimensional scalar wave equation of the form

$$
\begin{aligned}
\Gamma_{x} f_{x} & =\Lambda_{x} g_{x} \\
\Gamma_{y} f_{y} & =\Lambda_{y} g_{y} \\
\Gamma_{z} f_{z} & =\Lambda_{z} g_{z} \\
\Gamma_{x} g_{x} & =\Lambda_{x} f \\
\Gamma_{y} g_{y} & =\Lambda_{y} f \\
\Gamma_{z} g_{z} & =\Lambda_{z} f \\
f & =f_{x}+f_{y}+f_{z}
\end{aligned}
$$

the centered finite-difference system is simply obtained by replacing the operators $\Gamma_{u}$ and $\Lambda_{u}$ by their discrete counterparts

$$
\begin{aligned}
\Gamma_{u} & \Rightarrow \Delta_{t}-\sigma_{u} \Sigma_{t} \\
\Lambda_{u} & \Rightarrow c_{u} \Delta_{u}+\bar{\sigma}_{u} \Sigma_{u}
\end{aligned}
$$

Each equation has then the form of (44) and can be written in the form of (46) for direct computer implementation, the coefficients being determined using one of the prescriptions described in 3.1 .

\subsubsection{The vector three dimensional Maxwell equations}

We consider Maxwell equations written in normalized units

$$
\begin{aligned}
\frac{\partial \mathbf{B}}{\partial t} & =-\nabla \times \mathbf{E} \\
\frac{\partial \mathbf{E}}{\partial t} & =\nabla \times \mathbf{B}-\mathbf{J} \\
\nabla \cdot \mathbf{E} & =\rho \\
\nabla \cdot \mathbf{B} & =0
\end{aligned}
$$

which can also be expressed as

$$
\begin{aligned}
& \frac{\partial \mathbf{B}}{\partial t}=-\nabla \times \mathbf{E} \\
& \frac{\partial \mathbf{E}}{\partial t}=\nabla \times \mathbf{B}-\mathbf{J}+\nabla F
\end{aligned}
$$




$$
\begin{aligned}
\frac{\partial F}{\partial t} & =\nabla \cdot \mathbf{E}-\rho \\
\nabla \cdot \mathbf{B} & =0
\end{aligned}
$$

where $F \equiv 0$ in virtue of the equation of continuity $\frac{\partial \rho}{\partial t}+\nabla \cdot \mathbf{J}=0$.

We can derive from this a wave equation on the electric field

$$
\frac{\partial^{2} \mathbf{E}}{\partial t^{2}}-\Delta \mathbf{E}=\frac{\partial \mathbf{J}}{\partial t}-\nabla \rho
$$

A possible implementation consists of directly applying the algorithm described in 3.2.1 for the multidimensional scalar wave equation to each component of the electric field in (98)

$$
\begin{array}{lll}
\Gamma_{x} E_{x x}^{\prime}=\Lambda_{x} u_{x}-J_{x} & \Gamma_{x} u_{x}=\Lambda_{x} E_{x}-\rho \\
\Gamma_{y} E_{x y}^{\prime}=\Lambda_{y} u_{y} & \Gamma_{y} u_{y}=\Lambda_{y} E_{x} \\
\Gamma_{z} E_{x z}^{\prime}=\Lambda_{z} u_{z} & \Gamma_{z} u_{z}=\Lambda_{z} E_{x} \\
E_{x} & =E_{x x}^{\prime}+E_{x y}^{\prime}+E_{x z}^{\prime} & \\
\Gamma_{x} E_{y x}^{\prime}=\Lambda_{x} v_{x} & \Gamma_{x} v_{x}=\Lambda_{x} E_{y} \\
\Gamma_{y} E_{y y}^{\prime}=\Lambda_{y} v_{y}-J_{y} & \Gamma_{y} v_{y}=\Lambda_{y} E_{y}-\rho \\
\Gamma_{z} E_{y z}^{\prime}=\Lambda_{z} v_{z} & \Gamma_{z} v_{z}=\Lambda_{z} E_{y} \\
E_{y}=E_{y x}^{\prime}+E_{y y}^{\prime}+E_{y z}^{\prime} & & \\
\Gamma_{x} E_{z x}^{\prime}=\Lambda_{x} w_{x} & \Gamma_{x} w_{x}=\Lambda_{x} E_{z} \\
\Gamma_{y} E_{z y}^{\prime}=\Lambda_{y} w_{y} & \Gamma_{y} w_{y}=\Lambda_{y} E_{z} \\
\Gamma_{z} E_{z z}^{\prime}=\Lambda_{z} w_{z}-J_{z} & \Gamma_{z} w_{z}=\Lambda_{z} E_{z}-\rho \\
E_{z}=E_{z x}^{\prime}+E_{z y}^{\prime}+E_{z z}^{\prime} & &
\end{array}
$$

the magnetic field being given by

$$
\begin{aligned}
& B_{x}=v_{z}-w_{y} \\
& B_{y}=w_{x}-u_{z} \\
& B_{z}=u_{y}-v_{x}
\end{aligned}
$$

For completeness, the derivation of the explicit finite-difference discretization of (99) is given in Appendix C.

Using the formulation (97), an alternative implementation is given by

$$
\begin{aligned}
& \Gamma_{x} E_{x x}=\Lambda_{x} F-J_{x} \quad \Gamma_{x} F_{x}=\Lambda_{x} E_{x}-\rho_{x} \\
& \Gamma_{y} E_{x y}=\Lambda_{y} B_{z} \quad \Gamma_{y} B_{x y}=-\Lambda_{y} E_{z} \\
& \Gamma_{z} E_{x z}=-\Lambda_{z} B_{y} \quad \Gamma_{z} B_{x z}=\Lambda_{z} E_{y} \\
& E_{x} \quad=E_{x x}+E_{x y}+E_{x z} \quad B_{x} \quad=B_{x y}+B_{x z} \\
& \Gamma_{x} E_{y x}=-\Lambda_{x} B_{z} \quad \Gamma_{x} B_{y x}=\Lambda_{x} E_{z} \\
& \Gamma_{y} E_{y y}=\Lambda_{y} F-J_{y} \quad \Gamma_{y} F_{y}=\Lambda_{y} F-\rho_{y} \\
& \Gamma_{z} E_{y z}=\Lambda_{z} B_{x} \quad \Gamma_{z} B_{y z}=-\Lambda_{z} E_{x} \\
& E_{y} \quad=E_{y x}+E_{y y}+E_{y z} \quad B_{y} \quad=\quad B_{y x}+B_{y z} \\
& \Gamma_{x} E_{z x}=\Lambda_{x} B_{y} \quad \Gamma_{x} B_{z x}=-\Lambda_{x} E_{y} \\
& \Gamma_{y} E_{z y}=-\Lambda_{y} B_{x} \quad \Gamma_{y} B_{z y}=\Lambda_{y} E_{x} \\
& \Gamma_{z} E_{z z}=\Lambda_{z} F-J_{z} \quad \Gamma_{z} F_{z}=\Lambda_{z} E_{z}-\rho_{z} \\
& E_{z} \quad=E_{z x}+E_{z y}+E_{z z} \quad B_{z} \quad=\quad B_{z x}+B_{z y}
\end{aligned}
$$




$$
\begin{aligned}
\Gamma_{x} \rho_{x} & =-\Lambda_{x} J_{x} \\
\Gamma_{y} \rho_{y} & =-\Lambda_{y} J_{y} \\
\Gamma_{z} \rho_{z} & =-\Lambda_{z} J_{z} \\
\rho & =\rho_{x}+\rho_{y}+\rho_{z} \\
F & =F_{x}+F_{y}+F_{z}
\end{aligned}
$$

A third implementation may consist of using a split form of (98):

$$
\begin{aligned}
& \Gamma_{x} E_{x x}=-J_{x} \\
& \Gamma_{y} E_{x y}=\Lambda_{y} B_{z} \\
& \Gamma_{z} E_{x z}=-\Lambda_{z} B_{y} \\
& \Gamma_{y} B_{x y}=-\Lambda_{y} E_{z} \\
& E_{x}=E_{x x}+E_{x y}+E_{x z} \quad B_{x} \quad=B_{x y}+B_{x z} \\
& \Gamma_{x} E_{y x}=-\Lambda_{x} B_{z} \\
& \Gamma_{y} E_{y y}=-J_{y} \\
& \Gamma_{z} E_{y z}=\Lambda_{z} B_{x} \\
& \Gamma_{x} B_{y x}=\Lambda_{x} E_{z} \\
& E_{y}=E_{y x}+E_{y y}+E_{y z} \quad B_{y}=B_{y x}+B_{y z} \\
& \Gamma_{x} E_{z x}=\Lambda_{x} B_{y} \\
& \Gamma_{y} E_{z y}=-\Lambda_{y} B_{x} \\
& \Gamma_{z} E_{z z}=-J_{z} \\
& \Gamma_{x} B_{z x}=-\Lambda_{x} E_{y} \\
& \Gamma_{y} B_{z y}=\Lambda_{y} E_{x} \\
& E_{z}=E_{z x}+E_{z y}+E_{z z} \quad B_{z}=B_{z x}+B_{z y}
\end{aligned}
$$

Several considerations help us choose between these three implementations.

Remarking that

$$
\begin{aligned}
B_{x y} & =-w_{y} \\
B_{x z} & =v_{z} \\
B_{y x} & =w_{x} \\
B_{y z} & =-u_{z} \\
B_{z x} & =-v_{x} \\
B_{z y} & =u_{y} \\
F_{x} & =u_{x} \\
F_{y} & =v_{y} \\
F_{z} & =w_{z}
\end{aligned}
$$

it follows that the first and second implementations are equivalent.

Now, if we define the operator $\Gamma_{u}^{-1}$ such that

$$
\Gamma_{u}^{-1} \Gamma_{u} f=f
$$

then, using (105) to (112), we have 


$$
\begin{aligned}
\Gamma_{x} \Gamma_{y} \Gamma_{z} F & =\Gamma_{x} \Gamma_{y} \Gamma_{z}\left(F_{x}+F_{y}+F_{z}\right) \\
& =\Gamma_{y} \Gamma_{z}\left(\Lambda_{x} E_{x}-\rho_{x}\right)+\Gamma_{x} \Gamma_{z}\left(\Lambda_{y} E_{y}-\rho_{y}\right)+\Gamma_{x} \Gamma_{y}\left(\Lambda_{z} E_{z}-\rho_{z}\right) \\
& =\Lambda_{x}\left(\Gamma_{x}^{-1} \Gamma_{y} \Gamma_{z}\left(\Lambda_{x} F-J_{x}\right)+\Lambda_{y} \Gamma_{z} B_{z}-\Lambda_{z} \Gamma_{y} B_{y}\right)-\Gamma_{y} \Gamma_{z} \rho_{x} \\
& +\Lambda_{y}\left(-\Lambda_{x} \Gamma_{z} B_{z}+\Gamma_{y}^{-1} \Gamma_{x} \Gamma_{z}\left(\Lambda_{y} F-J_{y}\right)+\Lambda_{z} \Gamma_{x} B_{x}\right)-\Gamma_{y} \Gamma_{z} \rho_{x} \\
& +\Lambda_{z}\left(\Lambda_{x} \Gamma_{y} B_{y}-\Lambda_{y} \Gamma_{x} B_{x}+\Gamma_{z}^{-1} \Gamma_{x} \Gamma_{y}\left(\Lambda_{z} F-J_{z}\right)\right)-\Gamma_{y} \Gamma_{z} \rho_{x} \\
& =\left(\Lambda_{x}^{2} \Gamma_{x}^{-1} \Gamma_{y} \Gamma_{z}+\Lambda_{y}^{2} \Gamma_{y}^{-1} \Gamma_{x} \Gamma_{z}+\Lambda_{z}^{2} \Gamma_{z}^{-1} \Gamma_{x} \Gamma_{y}\right) F
\end{aligned}
$$

Hence, $F$ depends only on itself and not on any other term and remains zero if is zero at any given time. This demonstration is valid in the discrete space only if the discrete operators $\Lambda$ and $\Gamma$ retain their properties of associativity and commutativity. It can easily be shown that this is the case, and it follows that all three implementations are equivalent. We have verified this result numerically. In practice, the third one is preferred because it is more efficient.

\section{REFLECTION OF A PLANE WAVE STRIKING AN APML}

We assume that we solve the following system

$$
\begin{aligned}
F_{x, j k}^{i+1} & =\alpha_{j} F_{x, j k}^{i}+\beta_{p, j} G_{x, j+1 / 2 k}^{i+1 / 2}-\beta_{m, j} G_{x, j-1 / 2 k}^{i+1 / 2} \\
F_{y, j k}^{i+1} & =F_{y, j k}^{i+1}+\frac{c_{y} \delta t}{\delta y}\left(G_{y, j k+1 / 2}^{i+1 / 2}-G_{y, j k-1 / 2}^{i+1 / 2}\right) \\
F_{j k}^{i+1} & =F_{x, j k}^{i+1}+F_{y, j k}^{i+1} \\
G_{x, j+1 / 2 k}^{i+1 / 2} & =\alpha_{j+1 / 2} G_{x, j+1 / 2 k}^{i-1 / 2}+\beta_{p, j+1 / 2} F_{j+1 k}^{i}-\beta_{m, j+1 / 2} F_{j k}^{i} \\
G_{y, j+1 / 2 k}^{i+1 / 2} & =G_{y, j+1 / 2 k}^{i-1 / 2}+\frac{c_{y} \delta t}{\delta y}\left(F_{j k+1}^{i}-F_{j k}^{i}\right)
\end{aligned}
$$

on a 2 -D grid.

\subsection{Analytical evaluation of the coefficient of reflection}

We consider a plane wave whose axis of propagation is at angle $\varphi$ from the normal of the absorbing layer. As schematically shown on Fig.2, multiple reflections occur in an APML layer. The calculation of the coefficient of reflection for the entire layer requires the knowledge of the coefficients of reflection at each plane of the layer (locations $\mathrm{j}, \mathrm{j}+1 / 2, \mathrm{j}+1, \mathrm{j}+3 / 2$, etc.).

\subsubsection{Coefficient of reflection given by a plane passing through a row of nodes}

We first evaluate the coefficient of reflection at a row passing through a node (location of F) inside a slice where the APML scheme is applied. The rest of space is described by centered finite-difference of the wave equation in vacuum. Hence, only the plane where the APML scheme applies will generate reflections.

Combining equations from the system (127), we deduce that (using the notation $\nu_{u}=\frac{c_{u} \delta t}{\delta u}$ with $u=x, y$ ) 


$$
\begin{aligned}
F_{j k}^{i+1} & +F_{j k}^{i}\left(-2+2 \nu_{y}^{2}-\alpha+\beta_{p} \nu_{x}+\beta_{m} \nu_{x}\right) \\
& +F_{j k}^{i-1}\left(1+2 \alpha-2 \alpha \nu_{y}^{2}-\beta_{p} \nu_{x}-\beta_{m} \nu_{x}\right)-\alpha F_{j k}^{i-1} \\
& +\nu_{y}^{2}\left[-F_{j k+1}^{i}-F_{j k-1}^{i}+\alpha\left(F_{j k+1}^{i-1}+F_{j k-1}^{i-1}\right)\right] \\
& +\nu_{x}\left[\beta_{p}\left(F_{j+1}^{i-1}-F_{j+1}^{i}\right)+\beta_{m}\left(F_{j-1}^{i-1}-F_{j-1}^{i}\right)\right] \\
& =0
\end{aligned}
$$

We assume now a plane wave of amplitude $e^{i\left(\omega t-k_{x} x-k_{y} y\right)}$ striking the APML slice at incidence $\varphi\left(k_{x}=k \cos (\varphi), k_{y}=k \sin (\varphi)\right)$. We assume that the norms of $k_{x}$ and $k_{y}$ are conserved by the transmitted and the reflected waves and we define the coefficient of reflection to be the complex number $r$. Under these conditions, the transmitted wave is given by $(1-r) e^{i\left(\omega t-k_{x} x-k_{y} y\right)}$, while the signal in front of the slice is the sum of the incident wave and the reflected one, that is $e^{i\left(\omega t-k_{x} x-k_{y} y\right)}+$ $r e^{i\left(\omega t+k_{x} x-k_{y} y\right)}$. Assuming that the slice stands at $\mathrm{j}$, we have

$$
\begin{aligned}
F_{j k}^{i+1} & =(1-r) e^{i \omega \delta t} \\
F_{j k}^{i} & =1-r \\
F_{j k}^{i-1} & =(1-r) e^{-i \omega \delta t} \\
F_{j k}^{i-2} & =(1-r) e^{-2 i \omega \delta t} \\
F_{j k+1}^{i} & =(1-r) e^{-i k_{y} \delta y} \\
F_{j k-1}^{i} & =(1-r) e^{i k_{y} \delta y} \\
F_{j k+1}^{i-1} & =(1-r) e^{i\left(-\omega \delta t-k_{y} \delta y\right)} \\
F_{j k-1}^{i-1} & =(1-r) e^{i\left(-\omega \delta t+k_{y} \delta y\right)} \\
F_{j+1 k}^{i} & =(1-r) e^{-i k_{x} \delta x} \\
F_{j+1 k}^{i-1} & =(1-r) e^{i\left(-\omega \delta t-k_{x} \delta x\right)} \\
F_{j-1 k}^{i} & =e^{i k_{x} \delta x}+r e^{-i k_{x} \delta x} \\
F_{j-1 k}^{i+1} & =e^{i\left(\omega \delta t+k_{x} \delta x\right)}+r e^{i\left(\omega \delta t-k_{x} \delta x\right)}
\end{aligned}
$$

Substituting these into (128), we get

$$
r_{n o d e}^{+}=\frac{a+b e^{i k_{x} \delta x}}{a+b e^{-i k_{x} \delta x}}
$$

with

$$
\begin{aligned}
a & =e^{i \omega \delta t}+\left(-2+2 \nu_{y}^{2}-\alpha+\beta_{p} \nu_{x}+\beta_{m} \nu_{x}\right) \\
& +e^{-i \omega \delta t}\left(1+2 \alpha-2 \alpha \nu_{y}^{2}-\beta_{p} \nu_{x}-\beta_{m} \nu_{x}\right)-\alpha e^{-2 i \omega \delta t} \\
& +\nu_{y}^{2}\left(\alpha e^{-i \omega \delta t}-1\right)\left(e^{-i k_{y} \delta y}+e^{i k_{y} \delta y}\right) \\
& +\nu_{x} \beta_{p} e^{-i k_{x} \delta x}\left(1+e^{-i \omega \delta t}\right) \\
b & =\nu_{x} \beta_{m}\left(1-e^{-i \omega \delta t}\right)
\end{aligned}
$$


The calculation of $k_{x}$ and $k_{y}$ for a given $\omega$ is detailed in Appendix A.

The coefficient of reflection $r_{\text {node }}^{-}$for a wave propagating backward along $x$ is given by the same formula, switching $\beta_{p}$ and $\beta_{m}$.

\subsubsection{Coefficient of reflection given by a plane passing between two rows of nodes}

A the locations of $G_{x}$, we have

$$
G_{x, j+1 / 2 k}^{i+1 / 2}=\alpha G_{x, j+1 / 2 k}^{i-1 / 2}+\beta_{p} F_{j+1 k}^{i}-\beta_{m} F_{j k}^{i}
$$

Using the same considerations as for calculating the reflection at the node leads us to the coefficient of reflection at the internode in $x$

$$
r_{i}^{+}=\frac{a-b e^{i k_{x} \delta x}}{a+b e^{-i k_{x} \delta x}}
$$

with

$$
\begin{aligned}
a & =\left(e^{i \omega \delta t / 2}-\alpha e^{-i \omega \delta t / 2}\right) \cos (\varphi)+\beta_{p} e^{-i k_{x} \delta x / 2} \\
b & =\beta_{m}
\end{aligned}
$$

Again, the coefficient of reflection $r_{i}^{-}$for the wave propagating backward relative to $\mathrm{x}$, is obtained by switching $\beta_{p}$ and $\beta_{m}$. We also note that the knowledge of the coefficient of reflections $r^{+}$and $r^{-}$of one slice also gives us its coefficients of transmission $t^{+}=1-r^{+}$and $t^{-}=1-r^{-}$.

\subsubsection{Coefficient of reflection of the entire APML layer}

We assume that the APML layer lies from $j_{0}$ to $j_{0}+n_{L}$. Knowing the coefficients of reflection and transmission for both directions of two consecutive slices, say slices at $j_{0}+n_{L}-1 / 2$ and $j_{0}+n_{L}$, we can calculate the coefficient of reflection $R_{j_{0}-n_{L}-1 / 2}^{+}$ due to the coupling of these two slices (for clarity, we use the notation shortcut for indices: $1 \equiv j_{0}+n_{L}-1 / 2$ and $2 \equiv j_{0}+n_{L}$ )

$$
\begin{aligned}
R_{j_{0}+n_{L}-1 / 2}^{+}= & r_{1}^{+}-t_{1}^{+} r_{2}^{+} t_{1}^{-} e^{-i k_{x} \delta x}+t_{1}^{+} r_{2}^{+} t_{1}^{-} e^{-i k_{x} \delta x}\left(r_{1}^{-} r_{2}^{+} e^{-i k_{x} \delta x}\right) \\
& -t_{1}^{+} r_{2}^{+} t_{1}^{-} e^{-i k_{x} \delta x}\left(r_{1}^{-} r_{2}^{+} e^{-i k_{x} \delta x}\right)^{2} \ldots \\
= & r_{1}^{+}-t_{1}^{+} r_{2}^{+} t_{1}^{-} e^{-i k_{x} \delta x} \sum_{n=0}^{\infty}\left(-r_{1}^{-} r_{2}^{+} e^{-i k_{x} \delta x}\right)^{n} \\
= & r_{1}^{+}-\frac{t_{1}^{+} r_{2}^{+} t_{1}^{-} e^{-i k_{x} \delta x}}{1+r_{1}^{-} r_{2}^{+} e^{-i k_{x} \delta x}}
\end{aligned}
$$

We can iterate backward from $j=j_{0}+n_{L}$ to $j=j_{0}$ in order to get the coefficient of reflection of the entire layer, given by $R_{j_{0}}^{+}$, using at each iteration the formula

$$
R_{j}^{+}=r_{j}^{+}-\frac{t_{j}^{+} R_{j+1 / 2}^{+} t_{j}^{-} e^{-i k_{x} \delta x}}{1+r_{j}^{-} R_{j+1 / 2}^{+} e^{-i k_{x} \delta x}}
$$




\subsection{Evaluation of the coefficient of reflections via numerical experiments}

\subsubsection{In one dimension}

We compute the quantities

$$
\begin{aligned}
F_{j}^{i+1} & =\alpha_{j} F_{j}^{i}+\beta_{p, j} G_{j+1 / 2}^{i+1 / 2}-\beta_{m, j} G_{j-1 / 2}^{i+1 / 2} \\
G_{j+1 / 2}^{i+1 / 2} & =\alpha_{j+1 / 2} G_{j+1 / 2}^{i-1 / 2}+\beta_{p, j+1 / 2} F_{j+1}^{i}-\beta_{m, j+1 / 2} F_{j}^{i}
\end{aligned}
$$

on a 1-D grid of length $N_{x}+N_{A P M L}$. At the left end of the grid, the field is imposed to be $F(\omega, t)=H_{f}(t) \sin (\omega t)$ where $H_{f}(t)$ is the Harris function

$$
H_{f}(t)= \begin{cases}\frac{10-15 \cos (2 \pi c t / L)+6 \cos (4 \pi c t / L)-\cos (6 \pi c t / L)}{32} & \text { when } 0<t<L / c \\ 0 & \text { otherwise }\end{cases}
$$

where $L=N_{x}$ and $c$ is the speed of waves. For $j<N_{x}$, we model propagation in vacuum, so that we set $\alpha=1$ and $\beta_{p}=\beta_{m}=c \delta t / \delta x$. For $j \geq N_{x}$, the coefficients are set according to model either a PML or an APML medium. A reference solution is concurrently calculated on a $2 N_{x}$ grid simulating propagation in vacuum only:

$$
\begin{aligned}
F_{r e f, j}^{i+1} & =F_{r e f, j}^{i}+\frac{c \delta t}{\delta x}\left(G_{r e f, j+1 / 2}^{i+1 / 2}-G_{r e f, j-1 / 2}^{i+1 / 2}\right) \\
G_{r e f, j+1 / 2}^{i+1 / 2} & =G_{r e f, j+1 / 2}^{i-1 / 2}+\frac{c \delta t}{\delta x}\left(F_{r e f, j+1}^{i}-F_{r e f, j}^{i}\right)
\end{aligned}
$$

The time step is set to $\delta t=0.5 \delta x / c$ and the run is stopped when the time $t$ reaches $t=2 N_{x} \delta x / c$ (note that we use $\delta t=0.5 \delta x / c$ for convenience and because it is below the Courant limit up to dimension 3 , so that the results are valid in 1-D, $2-\mathrm{D}$ and 3-D). The coefficient of reflection is then computed as

$$
R(\omega)=\sqrt{\frac{\sum_{j \leq N_{x}}\left[\left(F-F_{r e f}\right)^{2}+\left(G-G_{r e f}\right)^{2}\right]}{\sum_{j>N_{x}}\left[F_{r e f}^{2}+G_{r e f}^{2}\right]}}
$$

\subsubsection{In two dimensions}

We consider the quantities

$$
\begin{aligned}
F_{x, j k}^{i+1} & =\alpha_{j} F_{x, j k}^{i}+\beta_{p, j} G_{x, j+1 / 2 k}^{i+1 / 2}-\beta_{m, j} G_{x, j-1 / 2 k}^{i+1 / 2} \\
F_{y, j k}^{i+1} & =F_{y, j k}^{i}+\frac{c \delta t}{\delta y}\left(G_{y, j k+1 / 2}^{i+1 / 2}-G_{y, j k-1 / 2}^{i+1 / 2}\right) \\
F_{j k}^{i+1} & =F_{x, j l}^{i+1}+F_{y, j l}^{i+1} \\
G_{x, j+1 / 2 k}^{i+1 / 2} & =\alpha_{j+1 / 2} G_{x, j+1 / 2 k}^{i-1 / 2}+\beta_{p, j+1 / 2} F_{x, j+1 k}^{i}-\beta_{m, j+1 / 2} F_{x, j k}^{i} \\
G_{y, j k+1 / 2}^{i+1 / 2} & =G_{y, j k+1 / 2}^{i-1 / 2}+\frac{c \delta t}{\delta y}\left(F_{y, j k+1}^{i}-F_{y, j k}^{i}\right)
\end{aligned}
$$


on two 2-D grids (see Fig. 3). The first, that we label "Main grid", is composed of a large vacuum region bounded along $x$ by two APML regions (at the lower end a large region to simulate vacuum, at the upper end the APML layer to be tested). A second grid, labeled "Secondary grid", is composed of a small vacuum area followed by a large APML region. Periodic boundary conditions are applied in the $y$ dimension on both grids. Both grids have the same mesh size $\delta x$ and $\delta y$ in each dimension and the same length $L_{y}=N_{y} \delta_{y}$ in $y$.

We fix $\delta x=1, \delta y=1, \delta t=0.5, \omega$ and $\varphi$. Using (157), $\delta y=1, \delta t=0.5, \omega, \varphi$ and the result given in Appendix A, we evaluate the discrete $k_{y}$. From this, we can compute the wavelength along y: $\lambda_{y}=2 \pi / k_{y}$. Because we want $\lambda_{y}=L_{y}=N_{y} \delta_{y}$, we take $N_{y}$ to be the integer part of $\lambda_{y} / \delta_{y}$ and recompute $\lambda_{y}=N_{y} \delta_{y}$ and $k_{y}=$ $2 \pi / \lambda_{y}$. From this, we recompute $\varphi$ as

$$
\varphi=\arcsin \left|\frac{c \delta t}{\delta y} \frac{e^{-0.5 i k_{y} \delta_{y}}-e^{0.5 i k_{y} \delta_{y}}}{e^{0.5 i \omega \delta t}-e^{-0.5 i \omega \delta t}}\right|
$$

We then evaluate $k_{x}$ using the method of Appendix A.

At the lower end in $x$ of the secondary grid, the field is imposed to be

$$
F_{s, k}^{i}= \begin{cases}\frac{10-15 \cos (2 \pi c t / L)+6 \cos (4 \pi c t / L)-\cos (6 \pi c t / L)}{32} \sin \left(\omega t-k_{y} y\right) & \text { if } 0<t<0.5 L / c \\ \sin \left(\omega t-k_{y} y\right) & \text { otherwise }\end{cases}
$$

with $y=k \delta y$ and $t=i \delta t$.

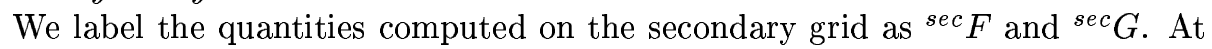
the lower end of the secondary grid, we impose

$$
{ }^{s e c} F_{0 k}^{i}=F_{s, k}^{i}
$$

and calculate the following ${ }^{\sec } G$ on the grid in $x$ :

$$
{ }^{s e c} G_{1 / 2 k}^{i+1 / 2}={ }^{s e c} G_{1 / 2 k}^{i-1 / 2}+\frac{c \delta t}{\delta x}\left({ }^{s e c} F_{1 k}^{i}-{ }^{s e c} F_{0 k}^{i}\right)
$$

The same field is also launched into the main grid in the vacuum region at location $j_{s}$, close to the APML region to be tested

$$
G_{x, j_{s}+1 / 2 k}^{i+1 / 2}=G_{x, j_{s}+1 / 2 k}^{i+1 / 2}+\frac{c \delta t}{\delta x}\left(F_{x, j_{s}+1 k}^{i}-F_{x, j_{s} k}^{i}-F_{s, k}^{i}\right)
$$

and we remove the contribution of the launched wave on $E$ at $j_{s}$

$$
F_{x, j_{s} k}^{i+1}=F_{x, j_{s} k}^{i}+\frac{c \delta t}{\delta x}\left(G_{x, j_{s}+1 / 2 k}^{i+1 / 2}-{ }^{s e c} G_{x, 1 / 2 k}^{i+1 / 2}-G_{x, j_{s}-1 / 2 k}^{i+1 / 2}\right)
$$

so that the incident wave does not affect the part at $j<j_{s}$ on the main grid. Hence, only the reflected wave is present in this region where it is measured at the lower end of the vacuum region.

\subsection{Results}

We compare the results we obtain using the APML technique with two implementations of the PML. The first one is the original implementation given by 
Berenger [2] (labeled "PML") while the second is one of our own [10] where we applied a steady state analysis in order to constrain the coefficients (labeled "PML- $\sigma$ adjusted").

In all the calculations, we have used

$$
\sigma_{j}=\sigma_{m}\left(\frac{j \delta x}{\Delta}\right)^{n} \quad 1 \leq j \leq N_{l}
$$

with $\sigma_{m}=4 / \delta x, \Delta=5 \delta x$ and $n=2$. The number of mesh points along $x$ in the layer was $N_{l}=10$. We also define the constant $p$ such that $\bar{\sigma}_{j}=p \sigma_{j}$. We have also set $c_{j}=c$ for any $j$. Given these prescriptions, a summary of the algorithms used is given in Appendix B.

For a given angular frequency $\omega$, the results are given as function of the vacum wavelength $\lambda=2 \pi c / \omega$ (where $c$ is the speed of wave in vacuum at the infinitesimal limit) and the mesh size $\delta x$, or the period $\tau=2 \pi / \omega$.

\subsubsection{In one dimension}

The analytical coefficients of reflection for the implementations APML-exponential, APML-SSA and APML-LWA are displayed for several values of $p$ in Figs. 4-6 respectively. We remark that the best result is always obtained for $p=-1$. We also notice that the implementation APML-LWA has the property of producing a coefficient of reflection that is independent of the value of $p$ or, in other words, independent of $\bar{\sigma}$. We recall that this is a property of the APML at the infinitesimal limit (see 2.2). Finally, we note that the APML-SSA implementation is equivalent to the APML-LWA implementation for $p=-1$.

On Fig. 7, we have plotted for comparison the coefficients of reflection given by the PML, PML- $\sigma$ adjusted, APML-Exponential ( $\mathrm{p}=-1)$, APML-Hybrid and APMLLWA. Both the analytical result and the results from the numerical experiment described in 4.2.1 are displayed. We remark first (as already noted in [10]) that our implementation of the PML (the PML- $\sigma$ adjusted) performs significantly better than the standard PML implementation. We remark also that all three APML implementations perform better than the standard PML, with the APML-Hybrid and the APML-LWA implementations performing the best.

\subsubsection{In two dimensions}

We display in Figs. 8-15 the coefficients of reflection for the different implementations of the PML and the APML as a function of the angle between the incident wave axis of propagation and the normal of the absorbing layer. As in the 1-D case, we remark that the results obtained for the APML-LWA is independent of $p$ and that APML-SSA $(p=-1)$ gives the same result as APML-LWA. The sharp "spikes" that are present in most of the figures are attributed to destructive interference inside the absorbing layer. A comparison of the different PML and APML is also given in Fig. 16 for $\tau=2 \pi / \omega \simeq 20 \delta x / c$. Results from both analytical and numerical experiment are presented for a wide range of angles and they match very well. Here again, we conclude that the PML-LWA implementation performs the best for the plane wave analysis that we have considered. 


\section{CONCLUSION}

From general considerations on explicit calculations of the wave equation in the discrete space, we have deduced a simple form of the equation that has led us to introduce additional terms. The analysis of the new equation shows that it describes an asymmetric Perfectly Matched Layer medium (thus labeled APML). The analysis of the new medium has shown that the absorption efficiency is equivalent to that of a PML at the infinitesimal limit. However, in the discrete space, several implementations were derived and the results have shown a significant improvement over a standard PML, both in one and two dimensions, when analysing the response of the considered PML and APML implementations to a monochromatic plane wave.

\section{FURTHER CONSIDERATIONS}

In real calculations, the structure of waves impinging on the absorbing layer are more complicated than the monochromatic plane wave considered here, and further studies are needed in order to fully compare the efficiency of APML with that of other techniques. Because different applications will involve different wave structures, the result may well be application dependent.

Also, some exploration of the properties and capabilities of the APML system has been left for future work. For example, in an APML medium, the velocity of waves can vary spatially. We note that various authors have already considered a progressive slow-down of the wave into sponge layers in order to prevent reflection $[4,5]$. Additional studies are needed to determine how the spatial variations of this velocity should be tailored and combined with the modulation of wave amplitude, as is possible with an APML, to improve the absorption efficiency of the layer at the discrete level.

Although the motivation for the introduction of the APML came from the analysis of a finite-difference formulation of the wave equation, and only a finite-difference implementation of the APML has been considered in this paper, there is nothing fundamental linking the APML formulation, as given in section 2.1, to the finitedifference technique. Hence, different discretization of the APML, such as schemes based on finite-element or finite-volume methods, are possible and may provide algorithms with interesting properties and performance.

Finally, like the original PML [2], the APML derivation that has been presented in this paper involves a splitting of field components and is thus only weakly wellposed [1,9]. Although, as mentioned in [9], instabilities due to the weakly well-posed property of the split-field formulation are not observable in most practical cases, an unsplit strongly well-posed formulation of the APML would be of interest (unsplit formulations of the PML can be found in $[3,7,9,13]$ ). As for the PML, such a formulation may permit the derivation of APML medium formulations for a variety of equations, broadening its range of application beyond electromagnetics to such fields as acoustics, quantum mechanics, and others.

\section{APPENDIX A: EVALUATION OF THE DISCRETE WAVE NUMBER}

Given the equation

$$
F_{j}^{i+1}=\alpha F_{j}^{i}+\beta_{p} G_{j+1 / 2}^{i+1 / 2}-\beta_{m} G_{j-1 / 2}^{i+1 / 2}
$$


supporting the propagation of a wave $e^{i(\omega t-k x)}$, we have

$$
e^{i \omega \delta t / 2}=\alpha e^{-i \omega \delta t / 2}+\beta_{p} e^{-i k \delta x / 2}-\beta_{m} e^{i k \delta x / 2}
$$

for a wave propagating in the forward direction. We suppose that $\omega$ is known and that we want to know the corresponding value of $k$. Multiplying the last equation by $e^{-i k \delta x / 2}$, we obtain a second order equation on $e^{-i k \delta x / 2}$

$$
\beta_{p}\left(e^{-i k \delta x / 2}\right)^{2}+\left(e^{i \omega \delta t / 2}-\alpha e^{-i \omega \delta t / 2}\right) e^{-i k \delta x / 2}-\beta_{m}=0
$$

that we can solve to get

$$
k=-2 \ln \left[\frac{-b+\sqrt{b^{2}-4 a c}}{2 a}\right]
$$

with

$$
\begin{aligned}
a & =\beta_{p} \\
b & =\left(e^{i \omega \delta t / 2}-\alpha e^{-i \omega \delta t / 2}\right) \\
c & =-\beta_{m}
\end{aligned}
$$

The solution for waves traveling backward is obtained by interchanging $\beta_{p}$ and $\beta_{m}$. In 2-D, we have

$$
\begin{aligned}
& F_{x, j k}^{i+1}=\alpha_{x} F_{x, j k}^{i}+\beta_{x, p} G_{x, j+1 / 2 k}^{i+1 / 2}-\beta_{x, m} G_{x j-1 / 2 k}^{i+1 / 2} \\
& F_{y, j k}^{i+1}=\alpha_{y} F_{y, j k}^{i}+\beta_{y, p} G_{y, j k+1 / 2}^{i+1 / 2}-\beta_{y, m} G_{y j k-1 / 2}^{i+1 / 2}
\end{aligned}
$$

Assuming $k_{x}=k \cos \varphi$ and $k_{y}=k \sin \varphi$, we get

$$
\begin{aligned}
e^{i \omega \delta t / 2} \cos \varphi & =\alpha_{x} e^{-i \omega \delta t / 2} \cos \varphi+\beta_{x, p} e^{-i k_{x} \delta x / 2}-\beta_{x, m} e^{i k_{x} \delta x / 2} \\
e^{i \omega \delta t / 2} \sin \varphi & =\alpha_{y} e^{-i \omega \delta t / 2} \sin \varphi+\beta_{y, p} e^{-i k_{y} \delta y / 2}-\beta_{y, m} e^{i k_{y} \delta y / 2}
\end{aligned}
$$

so that

$$
k_{x}=-2 \ln \left[\frac{-b_{x}+\sqrt{b_{x}^{2}-4 a_{x} c_{x}}}{2 a_{x}}\right]
$$

with

$$
\begin{aligned}
a_{x} & =\beta_{x, p} \\
b_{x} & =\left(e^{i \omega \delta t / 2}-\alpha e^{-i \omega \delta t / 2}\right) \cos \varphi \\
c_{x} & =-\beta_{x, m}
\end{aligned}
$$

and

$$
k_{y}=-2 \ln \left[\frac{-b_{y}+\sqrt{b_{y}^{2}-4 a_{y} c_{y}}}{2 a_{y}}\right]
$$


with

$$
\begin{aligned}
a_{y} & =\beta_{y, p} \\
b_{y} & =\left(e^{i \omega \delta t / 2}-\alpha e^{-i \omega \delta t / 2}\right) \sin \varphi \\
c_{y} & =-\beta_{y, m}
\end{aligned}
$$

\section{APPENDIX B: SUMMARY OF PML AND APML ALGORITHMS}

We assume $\sigma_{j}$ given at each location $j$.

$$
\begin{aligned}
F_{x, j k}^{i+1} & =\alpha_{j} F_{x, j k}^{i}+\beta_{p, j} G_{x, j+1 / 2 k}^{i+1 / 2}-\beta_{m, j} G_{x, j-1 / 2 k}^{i+1 / 2} \\
F_{y, j k}^{i+1} & =F_{y, j k}^{i}+\frac{c \delta t}{\delta y}\left(G_{y, j k+1 / 2}^{i+1 / 2}-G_{y, j k-1 / 2}^{i+1 / 2}\right) \\
F_{j k}^{i+1} & =F_{x, j k}^{i+1}+F_{y, j k}^{i+1} \\
G_{x, j+1 / 2 k}^{i+1 / 2} & =\alpha_{j+1 / 2} G_{x, j+1 / 2 k}^{i-1 / 2}+\beta_{p, j+1 / 2} F_{j+1 k}^{i}-\beta_{m, j+1 / 2} F_{j k}^{i} \\
G_{y, j k+1 / 2}^{i+1 / 2} & =G_{y, j k+1 / 2}^{i-1 / 2}+\frac{c \delta t}{\delta y}\left(F_{j k+1}^{i}-F_{j k}^{i}\right)
\end{aligned}
$$

\section{B.1. PML}

$$
\begin{aligned}
\alpha_{j} & =e^{-\sigma_{j} \delta t} \\
\beta_{p, j} & =\frac{1-e^{-\sigma_{j} \delta t}}{\sigma_{j} \delta x / c} \\
\beta_{m, j} & =\beta_{p, j}
\end{aligned}
$$

\section{B.2. PML- $\sigma$ adjusted}

$$
\begin{aligned}
\alpha_{j} & =e^{-\sigma_{j}^{*} \delta t} \\
\beta_{p, j} & =\frac{1-e^{-\sigma_{j}^{*} \delta t}}{\sigma_{j}^{*} \delta x / c} \\
\beta_{m, j} & =\beta_{p, j}
\end{aligned}
$$

with

$$
t_{j}=e^{-\sigma_{j} \delta x / 2}
$$

and

$$
\sigma_{j}^{*}=\frac{t_{j+1 / 2}-1 / t_{j}}{\delta x}
$$




\section{B.3. APML-Exponential}

$$
\begin{aligned}
\alpha_{j} & =e^{-\sigma_{j} \delta t} \\
\beta_{p, j} & =\frac{\bar{\sigma}_{j}}{\sigma_{j}} \frac{1-e^{-\sigma_{j} \delta t}}{1-e^{-\bar{\sigma}_{j} \delta x / c}} \\
\beta_{m, j} & =e^{-\bar{\sigma}_{j} \delta x / c} \beta_{p, j}
\end{aligned}
$$

with $\bar{\sigma}_{j}=p \sigma_{j}$

\section{B.4. APML-Hybrid}

$$
\begin{aligned}
\alpha_{j} & =1-\frac{c \delta t}{\delta x}\left[1+\left(\frac{\delta x-c \delta t}{\delta x+c \delta t}\right)\left(1-t_{j+1 / 2}\right)\right]+\frac{c \delta t}{\delta x} t_{j+1 / 2} \\
\beta_{p, j} & =\frac{c \delta t}{\delta x} \\
\beta_{m, j} & =\frac{c \delta t}{\delta x}\left[1+\left(\frac{\delta x-c \delta t}{\delta x+c \delta t}\right)\left(1-t_{j+1 / 2}\right)\right] t_{j}
\end{aligned}
$$

with

$$
t_{j}=e^{-\sigma_{j} \delta x / 2}
$$

\section{B.5. APML-SSA}

$$
\begin{aligned}
\alpha_{j} & =\frac{-1+\frac{\delta x}{c \delta t}\left(t_{p}+t_{m}+t_{p} t_{m}\left(t_{p p}+t_{m m}\right)\right)+t_{p} t_{m m} t_{p p} t_{m}}{1+\frac{\delta x}{c \delta t}\left(t_{p}+t_{m}+t_{p} t_{m}\left(t_{p p}+t_{m m}\right)\right)-t_{p} t_{m m} t_{p p} t_{m}} \\
\beta_{p, j} & =\frac{2 t_{m}\left(1+t_{m m} t_{p}\right)}{1+\frac{\delta x}{c \delta t}\left(t_{p}+t_{m}+t_{p} t_{m}\left(t_{p p}+t_{m m}\right)\right)-t_{p} t_{m m} t_{p p} t_{m}} \\
\beta_{m, j} & =\frac{2 t_{p}\left(1+t_{p p} t_{m}\right)}{1+\frac{\delta x}{c \delta t}\left(t_{p}+t_{m}+t_{p} t_{m}\left(t_{p p}+t_{m m}\right)\right)-t_{p} t_{m m} t_{p p} t_{m}}
\end{aligned}
$$

with $\bar{\sigma}_{j}=p \sigma_{j}$ and

$$
\begin{aligned}
t_{p} & =e^{-\left(\sigma_{j}+\bar{\sigma}_{j}\right) \delta x / 2} \\
t_{m m} & =e^{-\left(\sigma_{j}-\bar{\sigma}_{j}\right) \delta x / 2} \\
t_{p p} & =e^{-\left(\sigma_{j+1 / 2}+\bar{\sigma}_{j+1 / 2}\right) \delta x / 2} \\
t_{m} & =e^{-\left(\sigma_{j+1 / 2}-\bar{\sigma}_{j+1 / 2}\right) \delta x / 2}
\end{aligned}
$$

\section{B.6. APML-LWA}

$$
\alpha_{j}=\frac{-1+\gamma_{m}+t_{m} t_{p p}\left(\gamma_{p}+\gamma_{m}\right)+t_{p} t_{m m} t_{p p} t_{m}\left(\gamma_{p}+1\right)}{1+\gamma_{m}+t_{m} t_{p p}\left(\gamma_{p}+\gamma_{m}\right)+t_{p} t_{m m} t_{p p} t_{m}\left(\gamma_{p}-1\right)}
$$




$$
\begin{aligned}
\beta_{p, j} & =\frac{2 t_{m}\left(1+t_{m m} t_{p}\right)}{1+\gamma_{m}+t_{m} t_{p p}\left(\gamma_{p}+\gamma_{m}\right)+t_{p} t_{m m} t_{p p} t_{m}\left(\gamma_{p}-1\right)} \\
\beta_{m, j} & =\frac{2 t_{p}\left(1+t_{p p} t_{m}\right)}{1+\gamma_{m}+t_{m} t_{p p}\left(\gamma_{p}+\gamma_{m}\right)+t_{p} t_{m m} t_{p p} t_{m}\left(\gamma_{p}-1\right)}
\end{aligned}
$$

with $\bar{\sigma}_{j}=p \sigma_{j}, \gamma_{p}=\gamma_{m}=\delta x / c \delta t$ and

$$
\begin{aligned}
t_{p} & =e^{-\left(\sigma_{j}+\bar{\sigma}_{j}\right) \delta x / 2} \\
t_{m m} & =e^{-\left(\sigma_{j}-\bar{\sigma}_{j}\right) \delta x / 2} \\
t_{p p} & =e^{-\left(\sigma_{j+1 / 2}+\bar{\sigma}_{j+1 / 2}\right) \delta x / 2} \\
t_{m} & =e^{-\left(\sigma_{j+1 / 2}-\bar{\sigma}_{j+1 / 2}\right) \delta x / 2}
\end{aligned}
$$

\section{APPENDIX C: EXPLICIT FINITE-DIFFERENCE FORMULATION OF THE 3-D WAVE EQUATION}

Using (85) and (86), the system (99) rewrites

$$
\begin{array}{rlrl}
\left(\frac{\partial}{\partial t}-\sigma_{x}\right) E_{x x}^{\prime} & =\left(c_{x} \frac{\partial}{\partial x}-\bar{\sigma}_{x}\right) u_{x}-J_{x} & & \left(\frac{\partial}{\partial t}-\sigma_{x}\right) u_{x}=\left(c_{x} \frac{\partial}{\partial x}-\bar{\sigma}_{x}\right) E_{x}-\rho \\
\left(\frac{\partial}{\partial t}-\sigma_{y}\right) E_{x y}^{\prime} & =\left(c_{y} \frac{\partial}{\partial y}-\bar{\sigma}_{y}\right) u_{y} & \left(\frac{\partial}{\partial t}-\sigma_{y}\right) u_{y}=\left(c_{y} \frac{\partial}{\partial y}-\bar{\sigma}_{y}\right) E_{x} \\
\left(\frac{\partial}{\partial t}-\sigma_{z}\right) E_{x z}^{\prime} & =\left(c_{z} \frac{\partial}{\partial z}-\bar{\sigma}_{z}\right) u_{z} & & \left(\frac{\partial}{\partial t}-\sigma_{z}\right) u_{z}=\left(c_{z} \frac{\partial}{\partial z}-\bar{\sigma}_{z}\right) E_{x} \\
E_{x} & =E_{x x}^{\prime}+E_{x y}^{\prime}+E_{x z}^{\prime} & &
\end{array}
$$

The discretization using (94) and (95) together with the definitions (1), (2), (3) and (4) gives (without loss of generality, we consider the algorithm for given time step $\mathrm{i}$ and location $(\mathrm{j}, \mathrm{k}, \mathrm{l})$, dropping thus the indices $\mathrm{i}, \mathrm{j}, \mathrm{k}$ and $\mathrm{l}$ for a more concise 
presentation)

$$
\begin{aligned}
& \left(E_{x x 1 / 2,0,0}^{\prime 1 / 2}-E_{x x 1 / 2,0,0}^{\prime-1 / 2}\right) / \delta t=\sigma_{x 1 / 2,0,0} \quad\left(E_{x x 1 / 2,0,0}^{1 / 2}+E_{x x 1 / 2,0,0}^{\prime-1 / 2}\right) / 2 \\
& +c_{x 1 / 2,0,0} \quad\left(u_{x 1,0,0}^{0}-u_{x 0,0,0}^{0}\right) / \delta x \\
& +\bar{\sigma}_{x 1 / 2,0,0} \quad\left(u_{x 1,0,0}^{0}+u_{x 0,0,0}^{0}\right) / 2 \\
& -J_{x 1 / 2,0,0}^{0} \\
& \left(E_{x y 1 / 2,0,0}^{1 / 2}-E_{x y 1 / 2,0,0}^{\prime-1 / 2}\right) / \delta t=\sigma_{y 1 / 2,0,0} \quad\left(E_{x y 1 / 2,0,0}^{1 / 2}+E_{x y 1 / 2,0,0}^{\prime-1 / 2}\right) / 2 \\
& +c_{y 1 / 2,0,0} \quad\left(u_{y 1 / 2,1 / 2,0}^{0}-u_{y 1 / 2,-1 / 2,0}^{0}\right) / \delta y \\
& \bar{\sigma}_{y 1 / 2,0,0} \quad\left(u_{y 1 / 2,1 / 2,0}^{0}+u_{y 1 / 2,-1 / 2,0}^{0}\right) / 2 \\
& \left(E_{x z 1 / 2,0,0}^{1 / 2}-E_{x z 1 / 2,0,0}^{-1 / 2}\right) / \delta t=\sigma_{z 1 / 2,0,0} \quad\left(E_{x z 1 / 2,0,0}^{1 / 2}+E_{x z 1 / 2,0,0}^{\prime-1 / 2}\right) / 2 \\
& +c_{z 1 / 2,0,0} \quad\left(u_{z 1 / 2,0,1 / 2}^{0}-u_{z 1 / 2,0,-1 / 2}^{0}\right) / \delta z \\
& +\bar{\sigma}_{z 1 / 2,0,0} \quad\left(u_{z 1 / 2,0,1 / 2}^{0}+u_{z 1 / 2,0,-1 / 2}^{0}\right) / 2 \\
& \left(u_{x 0,0,0}^{1}-u_{x 0,0,0}^{0}\right) / \delta t \quad=\sigma_{x 0,0,0} \quad\left(u_{x 0,0,0}^{1}+u_{x 0,0,0}^{0}\right) / 2 \\
& +c_{x 0,0,0} \quad\left(E_{x 1 / 2,0,0}^{1 / 2}-E_{x-1 / 2,0,0}^{1 / 2}\right) \delta x \\
& +\bar{\sigma}_{x 0,0,0} \quad\left(E_{x 1 / 2,0,0}^{1 / 2}+E_{x-1 / 2,0,0}^{1 / 2}\right) / 2 \\
& -\quad \rho_{0,0,0}^{1 / 2} \\
& \left(u_{y 1 / 2,1 / 2,0}^{1}-u_{y 1 / 2,1 / 2,0}^{0}\right) / \delta t=\sigma_{y 1 / 2,1 / 2,0}\left(u_{y 1 / 2,1 / 2,0}^{1}+u_{y 1 / 2,1 / 2,0}^{0}\right) / 2 \\
& +c_{y 1 / 2,1 / 2,0}\left(E_{x 1 / 2,1,0}^{1 / 2}-E_{x 1 / 2,0,0}^{1 / 2}\right) / \delta y \\
& +\bar{\sigma}_{y 1 / 2,1 / 2,0}\left(E_{x 1 / 2,1,0}^{1 / 2}+E_{x 1 / 2,0,0}^{1 / 2}\right) / 2 \\
& \left(u_{z 1 / 2,0,1 / 2}^{1}-u_{z 1 / 2,0,1 / 2}^{0}\right) / \delta t=\sigma_{z 1 / 2,0,1 / 2}\left(u_{z 1 / 2,0,1 / 2}^{1}+u_{z 1 / 2,0,1 / 2}^{0}\right) / 2 \\
& +c_{z 1 / 2,0,1 / 2}\left(E_{x 1 / 2,0,1}^{1 / 2}-E_{x 1 / 2,0,0}^{1 / 2}\right) / \delta z \\
& +\bar{\sigma}_{z 1 / 2,0,1 / 2}\left(E_{x 1 / 2,0,1}^{1 / 2}+E_{x 1 / 2,0,0}^{1 / 2}\right) / 2
\end{aligned}
$$

with

$$
E_{x 1 / 2,0,0}^{1 / 2}=E_{x x 1 / 2,0,0}^{\prime 1 / 2}+E_{x y 1 / 2,0,0}^{\prime 1 / 2}+E_{x x 1 / 2,0,0}^{\prime 1 / 2}
$$

This gives the computer programmable form 


$$
\begin{aligned}
& E_{x x 1 / 2,0,0}^{\prime 1 / 2}=\alpha_{x 1 / 2,0,0} \quad E_{x x 1 / 2,0,0}^{\prime-1 / 2} \\
& +\beta_{p x 1 / 2,0,0} \quad u_{x 1,0,0}^{0} \\
& \text { - } \beta_{m x 1 / 2,0,0} \quad u_{x 0,0,0}^{0} \\
& -\gamma_{x 1 / 2,0,0} \quad J_{x 1 / 2,0,0}^{0} \\
& E_{x y 1 / 2,0,0}^{1 / 2}=\alpha_{y 1 / 2,0,0} \quad E_{x y 1 / 2,0,0}^{\prime-1 / 2} \\
& +\beta_{p y 1 / 2,0,0} \quad u_{y 1 / 2,1 / 2,0}^{0} \\
& \text { - } \beta_{m y 1 / 2,0,0} \quad u_{y 1 / 2,-1 / 2,0}^{0} \\
& E_{x z 1 / 2,0,0}^{1 / 2}=\alpha_{z 1 / 2,0,0} \quad E_{x z 1 / 2,0,0}^{\prime-1 / 2} \\
& +\beta_{p z 1 / 2,0,0} \quad u_{z 1 / 2,0,1 / 2}^{0} \\
& \text { - } \beta_{m z 1 / 2,0,0} \quad u_{z 1 / 2,0,-1 / 2}^{0} \\
& u_{x 0,0,0}^{1}=\alpha_{x 0,0,0} \quad u_{x 0,0,0}^{0} \\
& +\beta_{p x 0,0,0} \quad E_{x 1 / 2,0,0}^{1 / 2} \\
& \text { - } \beta_{m x 0,0,0} \quad E_{x-1 / 2,0,0}^{1 / 2} \\
& -\gamma_{x 0,0,0} \quad \rho_{0,0,0}^{1 / 2} \\
& u_{y 1 / 2,1 / 2,0}^{1}=\alpha_{y 1 / 2,1 / 2,0} \quad u_{y 1 / 2,1 / 2,0}^{0} \\
& +\beta_{p y 1 / 2,1 / 2,0} \quad E_{x 1 / 2,1,0}^{1 / 2} \\
& \text { - } \beta_{m y 1 / 2,1 / 2,0} \quad E_{x 1 / 2,0,0}^{1 / 2} \\
& u_{z 1 / 2,0,1 / 2}^{1}=\alpha_{z 1 / 2,0,1 / 2} \quad u_{z 1 / 2,0,1 / 2}^{0} \\
& +\beta_{p z 1 / 2,0,1 / 2} \quad E_{x 1 / 2,0,1}^{1 / 2} \\
& -\beta_{m z 1 / 2,0,1 / 2} \quad E_{x 1 / 2,0,0}^{1 / 2}
\end{aligned}
$$

where, for a given location $(\mathrm{j}, \mathrm{k}, \mathrm{l})$ and an axis $u=\{x, y$ or $z\}$, the equivalence between $\alpha_{u j, k, l}, \beta_{p u j, k, l}, \beta_{m u j, k, l}, \gamma_{u j, k, l}$ and $\sigma_{u j, k, l}, c_{u j, k, l}$ and $\bar{\sigma}_{u j, k, l}$ is given by

$$
\begin{aligned}
& \alpha_{u j, k, l}=\left(1-\sigma_{u j, k, l} \delta t / 2\right) /\left(1+\sigma_{u j, k, l} \delta t / 2\right) \\
& \beta_{p u j, k, l}=\delta t\left(1 / \delta u+\bar{\sigma}_{u j, k, l} / 2\right) /\left(1+\sigma_{u j, k, l} \delta t / 2\right) \\
& \beta_{m u j, k, l}=\delta t\left(1 / \delta u-\bar{\sigma}_{u j, k, l} / 2\right) /\left(1+\sigma_{u j, k, l} \delta t / 2\right) \\
& \gamma_{u j, k, l}=\delta t /\left(1+\sigma_{u j, k, l} \delta t / 2\right)=0.5 \delta t /\left(1+\alpha_{u j, k, l}\right)
\end{aligned}
$$

The values of $\alpha_{u j, k, l}, \beta_{p u j, k, l}, \beta_{m u j, k, l}$ are obtained from one of the formulas summarized in Appendix B.

\section{REFERENCES}

[1] S. Abarbanel, D. Gottlieb, A Mathematical Analysis of the PML Method, J. Comput. Phys. 134, 357 (1997).

[2] J.-P. Berenger, A Perfectly Matched Layer for the Absorption of Electromagnetic Waves, J. Comput. Phys. 114, 185 (1994). 
[3] F. Collino, P. Monk, Optimizing the Perfectly Matched Layer Computer Methods in Applied Mechanics and Engineering, Comput. Methods Appl. Mech. Engrg. 164, 157 (1998).

[4] M. Israeli, S. A. Orszag, Approximation of Radiation Boundary Conditions, J. Comput. Phys. 41, 115 (1981).

[5] S. Karni, Far-field Filtering Operators for Suppression of Reflections from Artificial Boundaries, SIAM J. Numer. Anal. 33, No. 3, 1014 (1996).

[6] A. Taflove, The Finite-Difference Time-Domain Method (Artech House, Norwood, MA, 1995).

[7] A. Taflove, Advances in Computational Electrodynamics: The FiniteDifference Time-Domain Method (Artech House, Norwood, MA, 1998).

[8] S. V. Tsynkov, Numerical Solution of Problems on Unbounded Domains. A Review., Applied Numerical Mathematics 27, 465 (1998).

[9] E. Turkel, A. Yefet, Absorbing PML Boundary Layers for Wave-like Equations, Applied Numerical mathematics 27, 533 (1998).

[10] J.-L. Vay, A New Absorbing Layer Boundary Condition for the Wave Equation, J. Comput. Phys. 165, 511 (2000).

[11] J.-L. Vay, An Extended FDTD Scheme for the Wave Equation. Application to Multiscale Electromagnetic Simulation, J. Comput. Phys. 167, 72 (2001).

[12] K. S. Yee, Numerical Solution of Initial Boundary Value Problems involving Maxwell's Equations in Isotropic Media, IEEE Trans. Antennas Propa. 14, 302 (1966).

[13] R. W. Ziolkowski, Time-Derivative Lorentz Material Model-Based Absorbing Boundary Conditions, IEEE Trans. on Antennas and Propagation 45, 1530 (1997) 


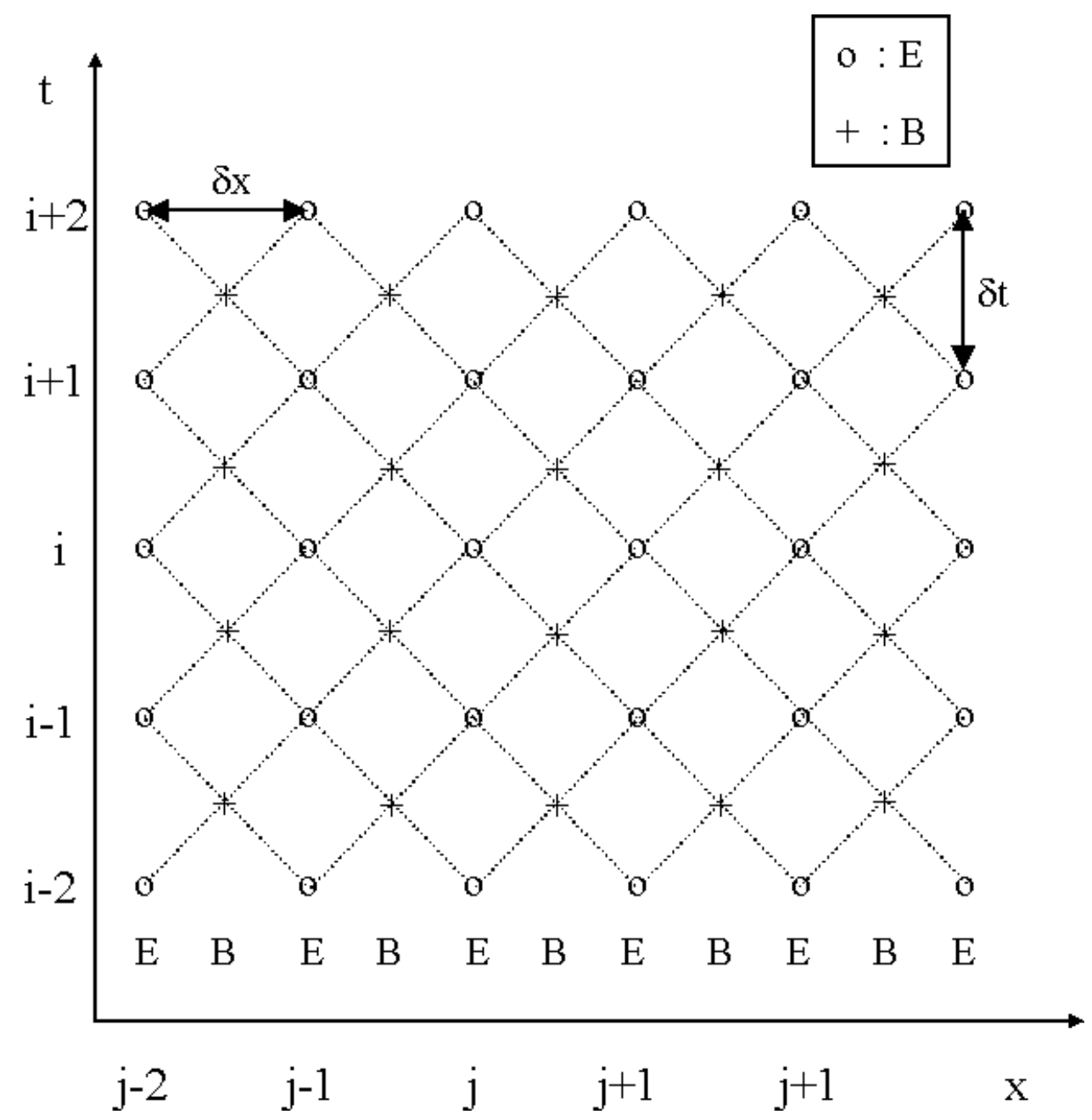

FIG. 1 Diagram showing the positions of E and B on the discrete space-time grid. $\mathrm{E}$ and $\mathrm{B}$ are both staggered in space and time. 


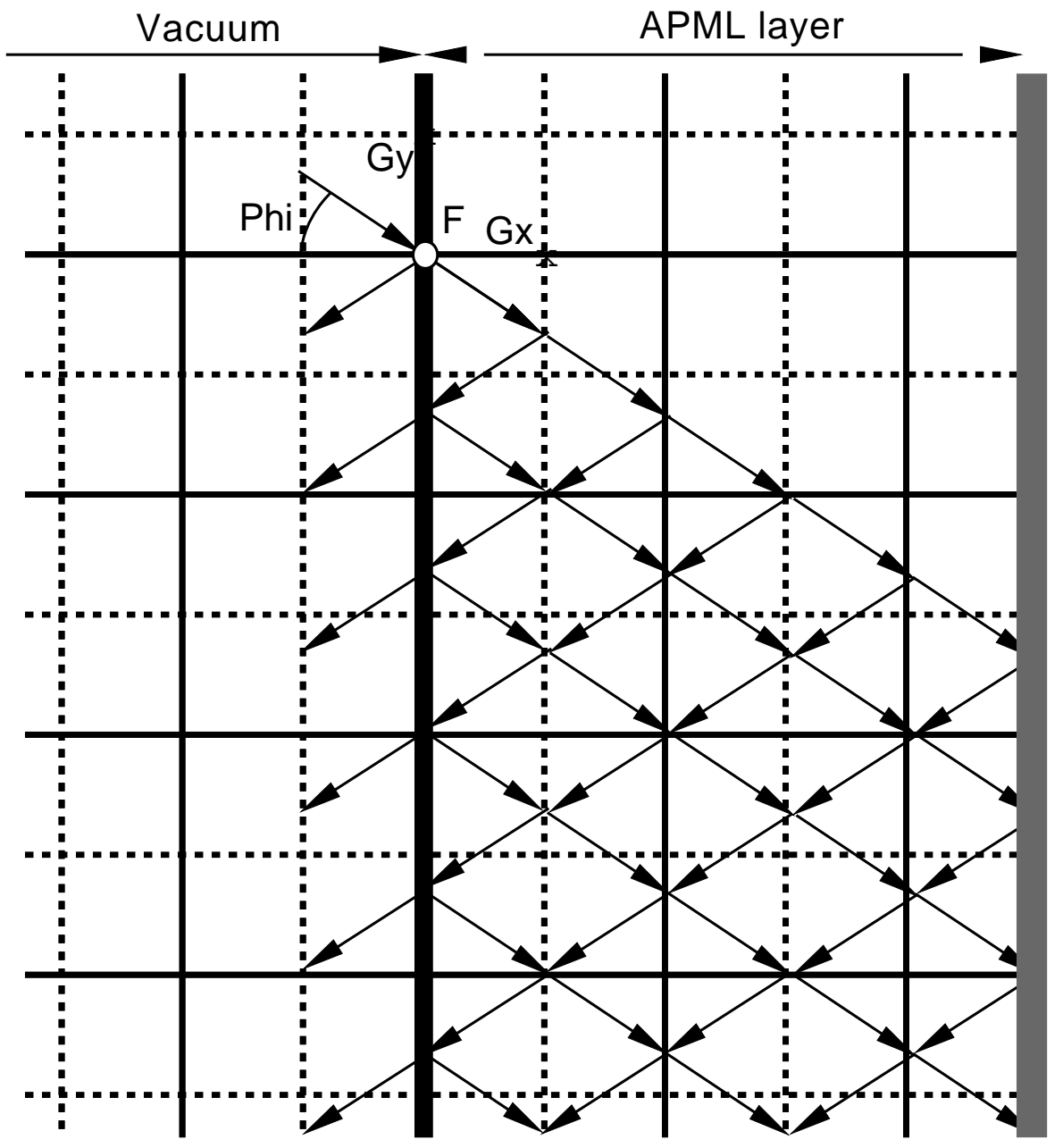

FIG. 2 A plane wave striking a APML layer will generate multiple reflections inside the layer that must be taken into account in order to calculate the coefficient of reflection. 


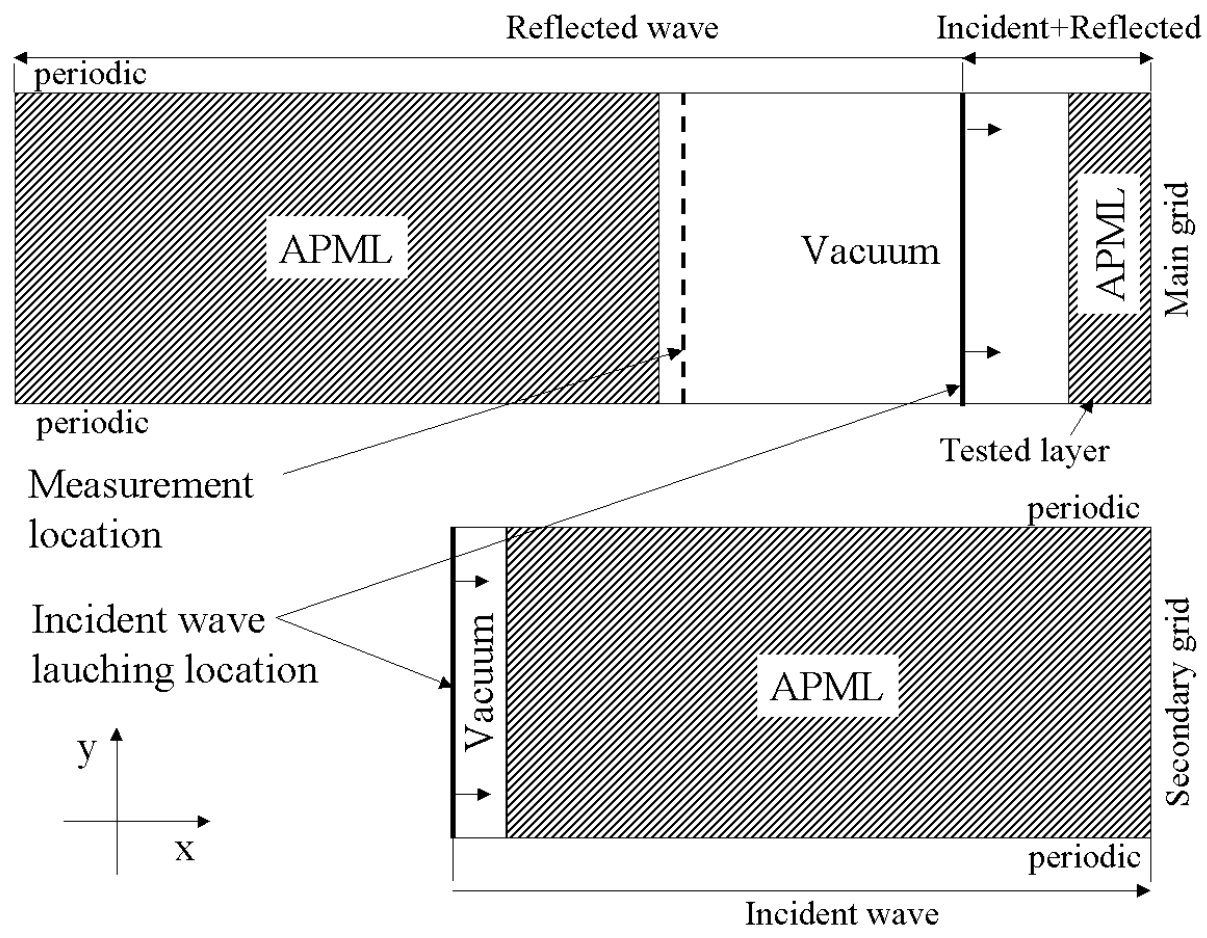

FIG. 3 Diagram of the "Main grid" and "Secondary grid" used for the measurement of the coefficient of reflection in two dimensions. 


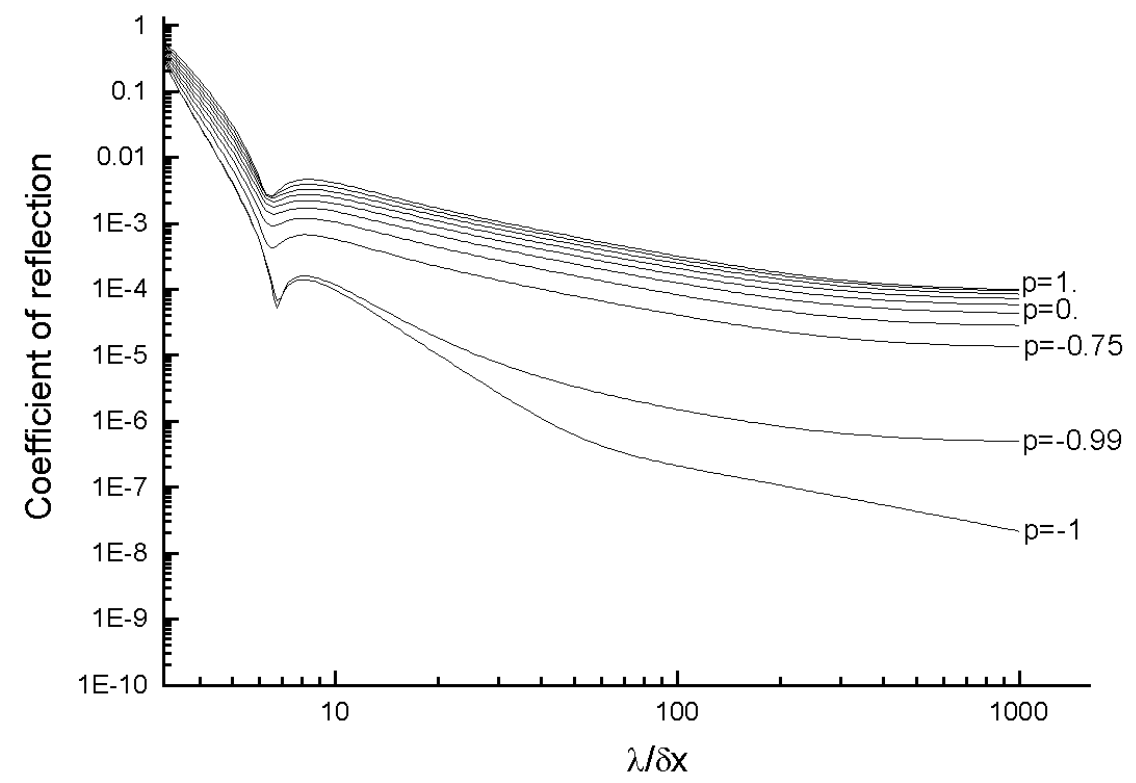

FIG. 4 Coefficient of reflection of the APML-Exponential as a function of wavelength for various values of $\mathrm{p}$. 


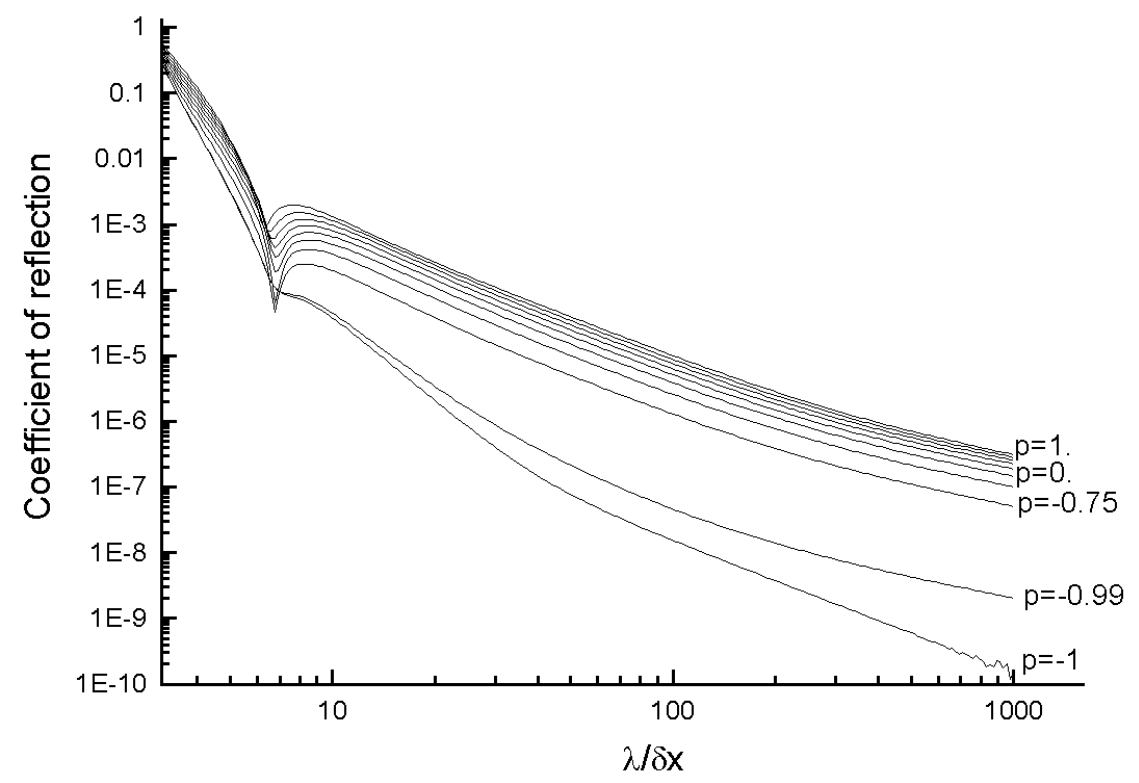

FIG. 5 Coefficient of reflection of the APML-SSA as a function of wavelength for various values of $p$. 


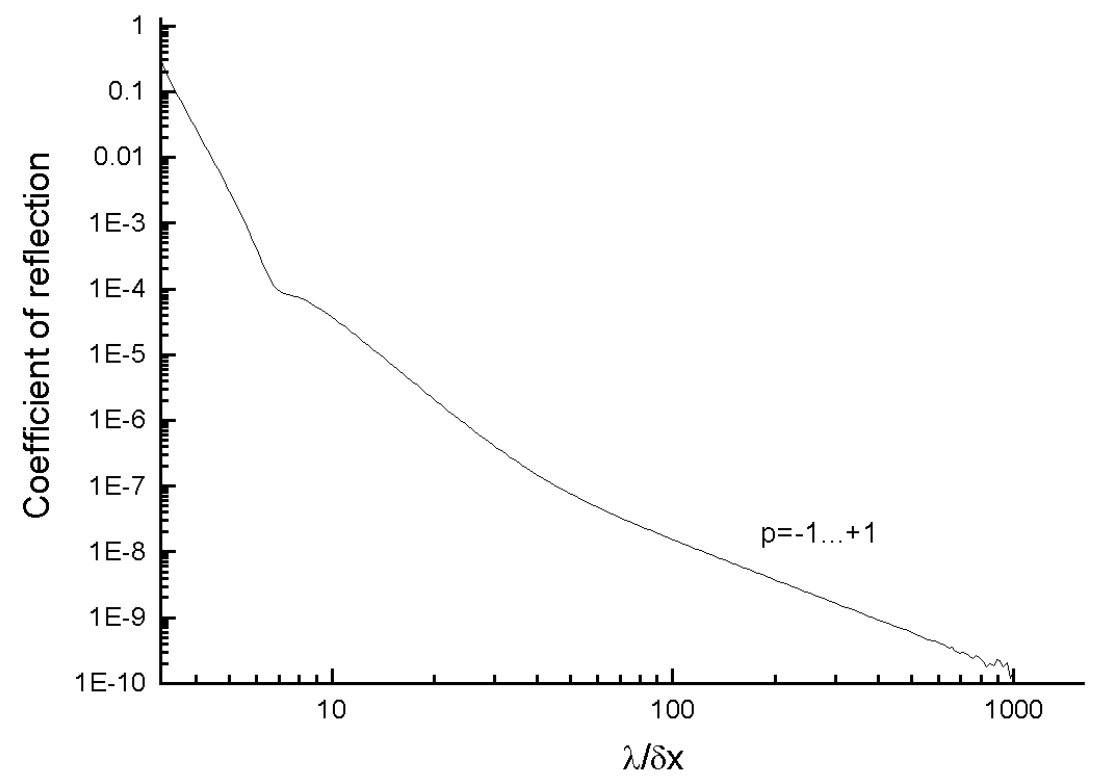

FIG. 6 Coefficient of reflection of the APML-LWA as a function of wavelength for various values of $\mathrm{p}$. 


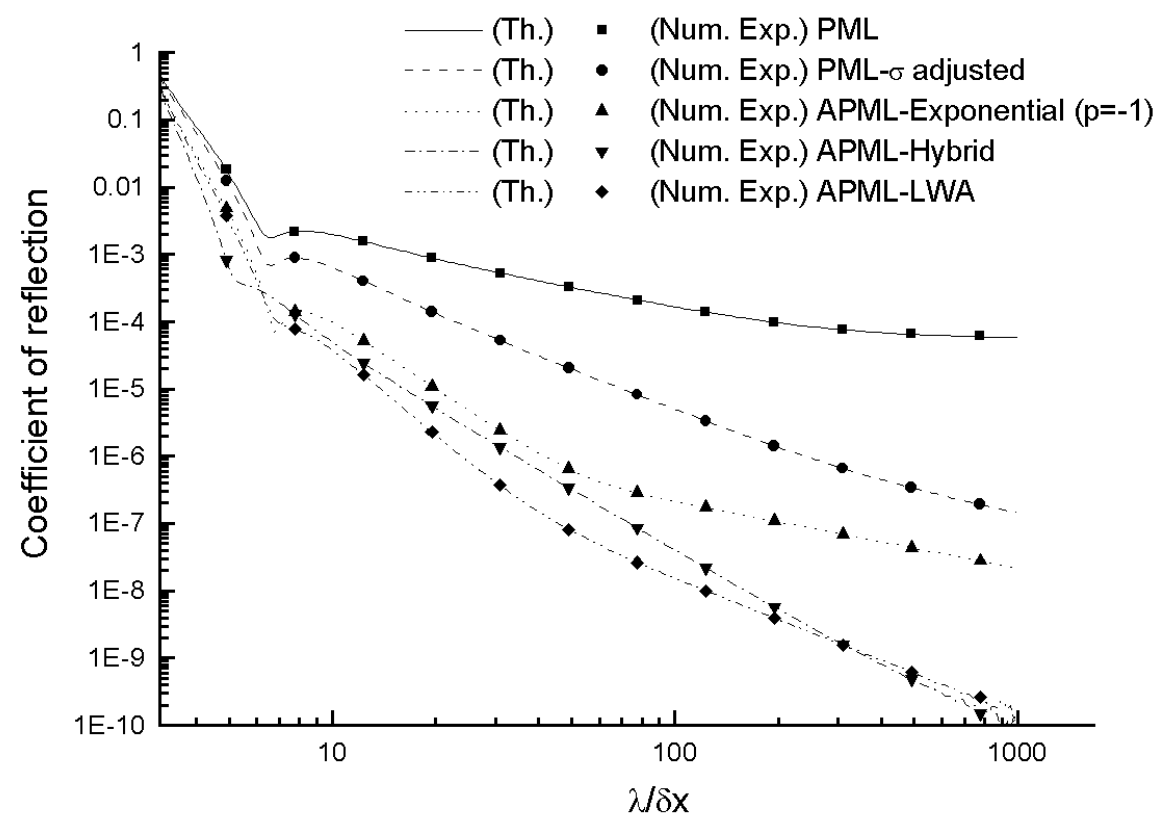

FIG. 7 Comparison of APML and PML coefficient of reflection as a function of wavelength in 1-D. 


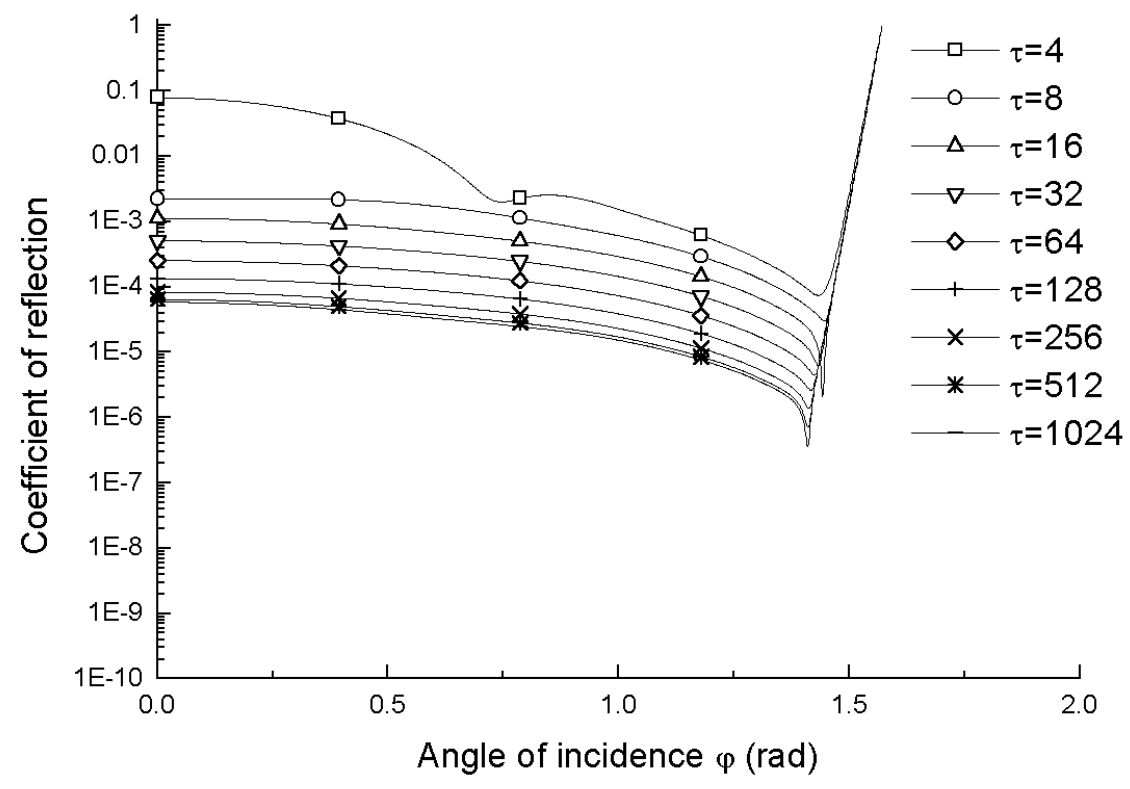

FIG. 8 Coefficient of reflection of the PML as a function of the angle of incidence. 


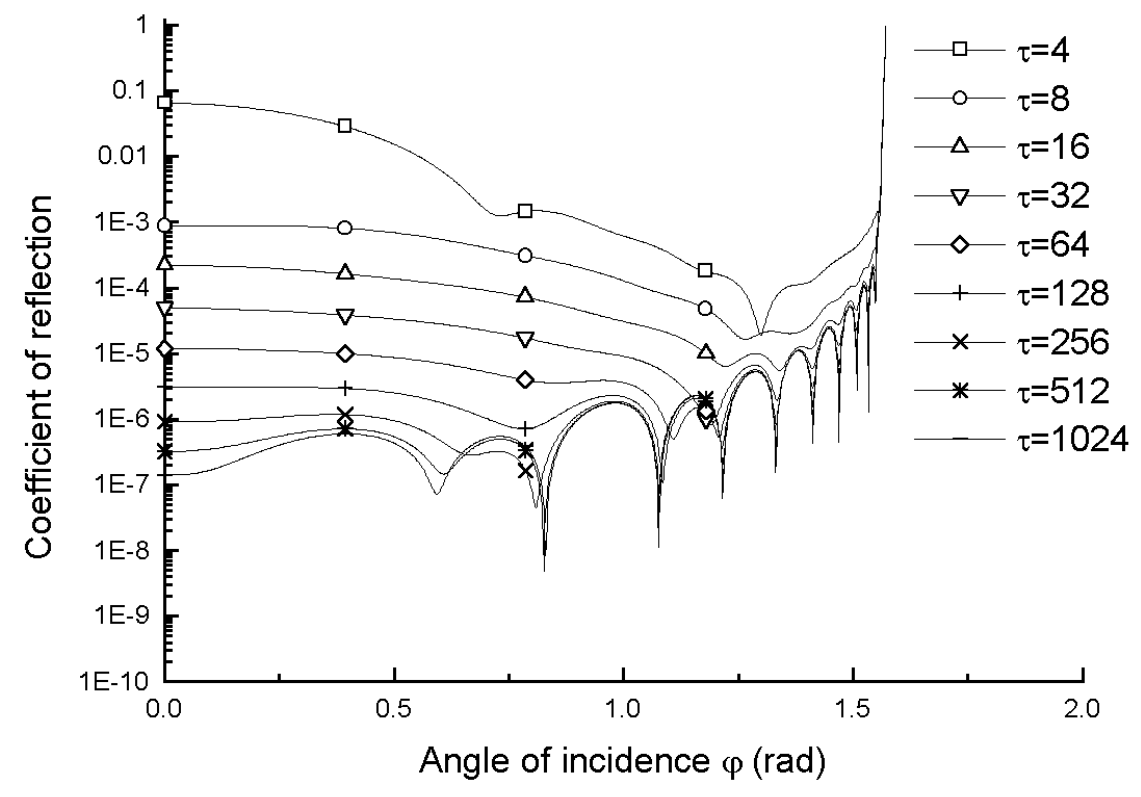

FIG. 9 Coefficient of reflection of the PML-sigma adjusted as a function of the angle of incidence. 


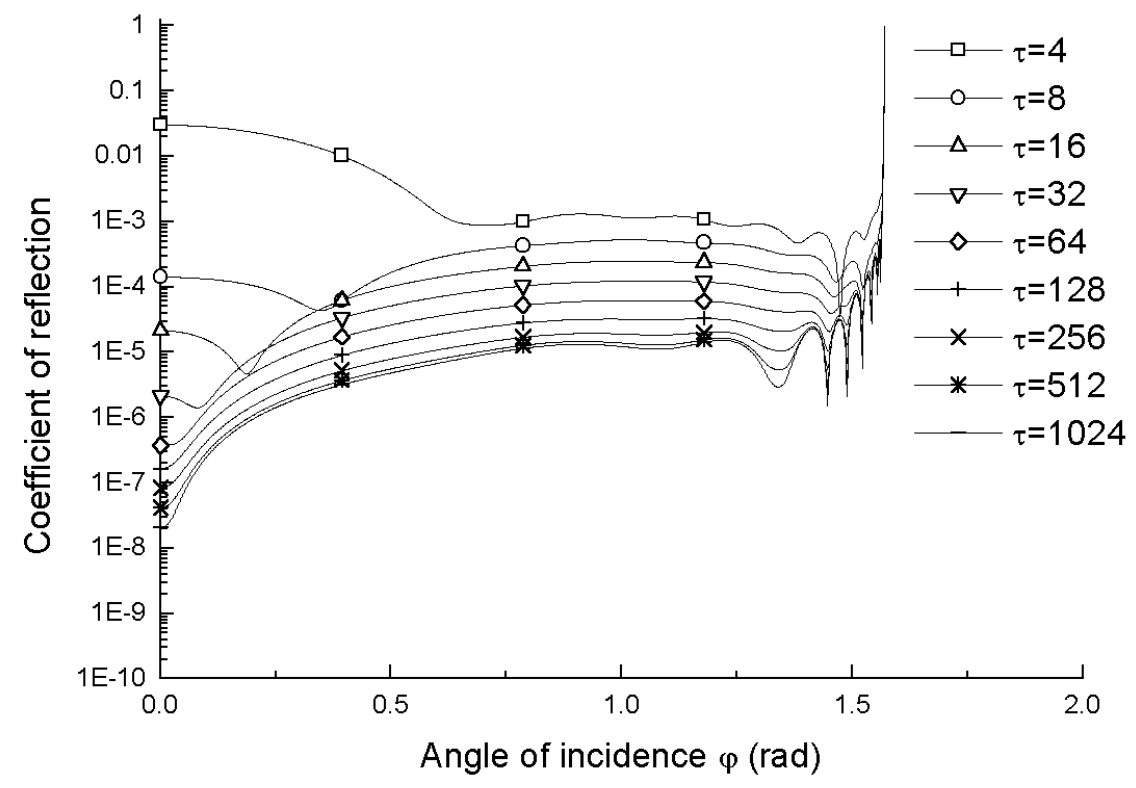

FIG. 10 Coefficient of reflection of the APML-exponential $(\mathrm{p}=-1)$ as a function of the angle of incidence. 


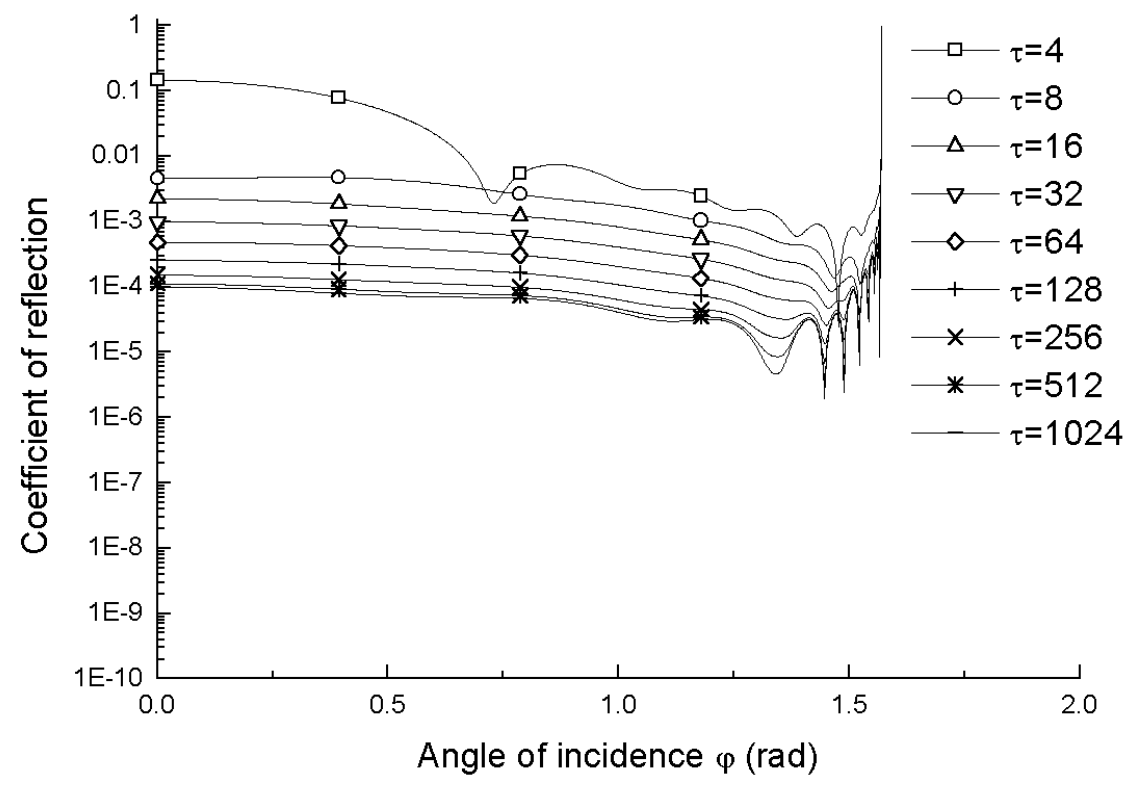

FIG. 11 Coefficient of reflection of the APML-exponential $(p=1)$ as a function of the angle of incidence. 


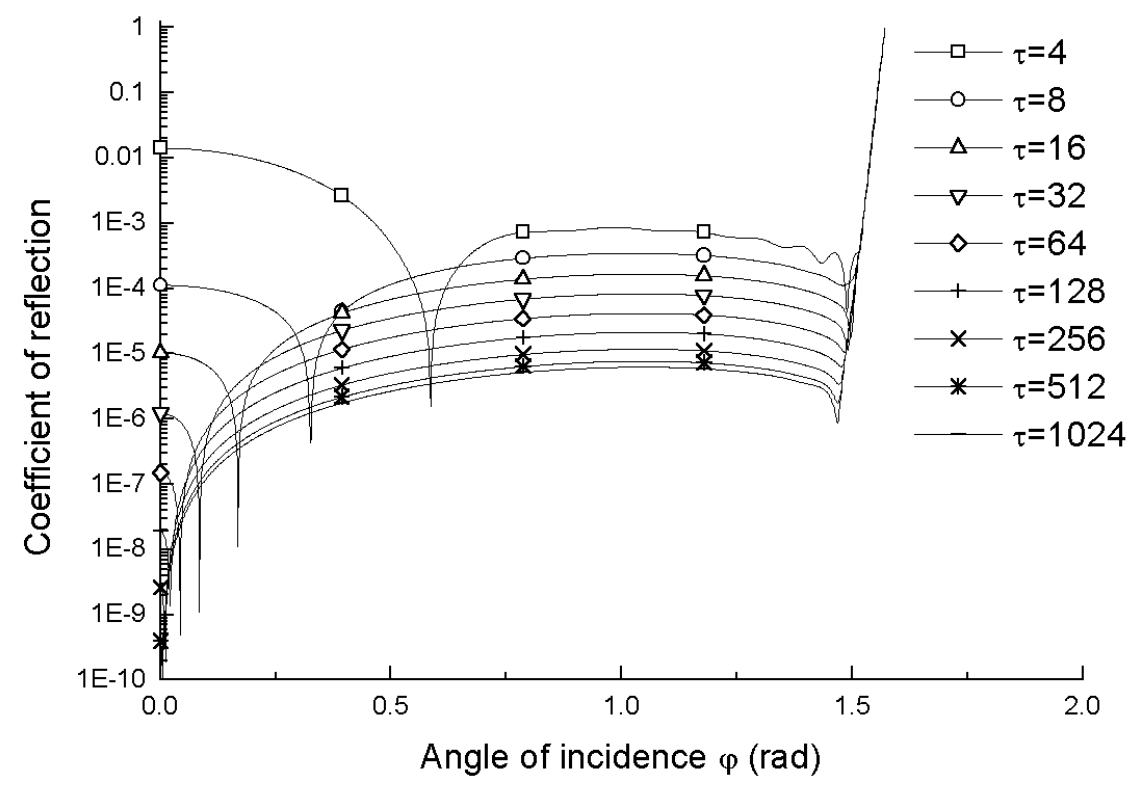

FIG. 12 Coefficient of reflection of the APML-Hybrid as a function of the angle of incidence. 


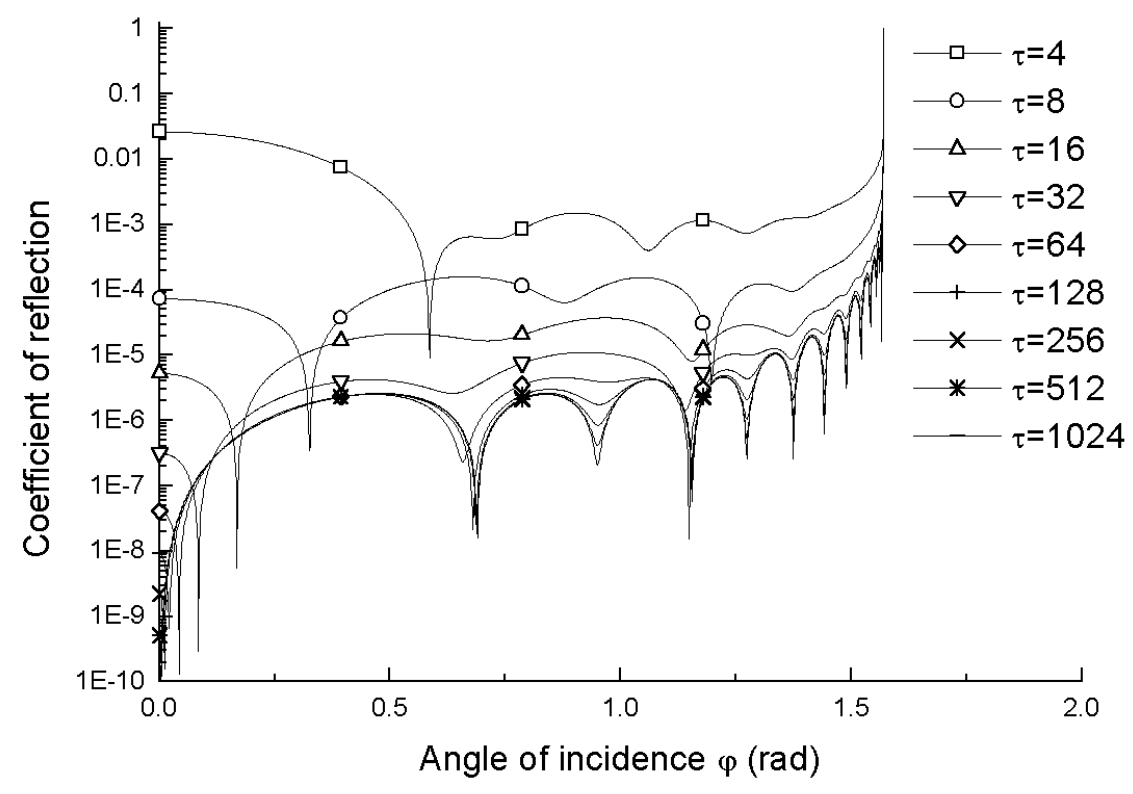

FIG. 13 Coefficient of reflection of the APML-SSA ( $p=-1)$ as a function of the angle of incidence. 


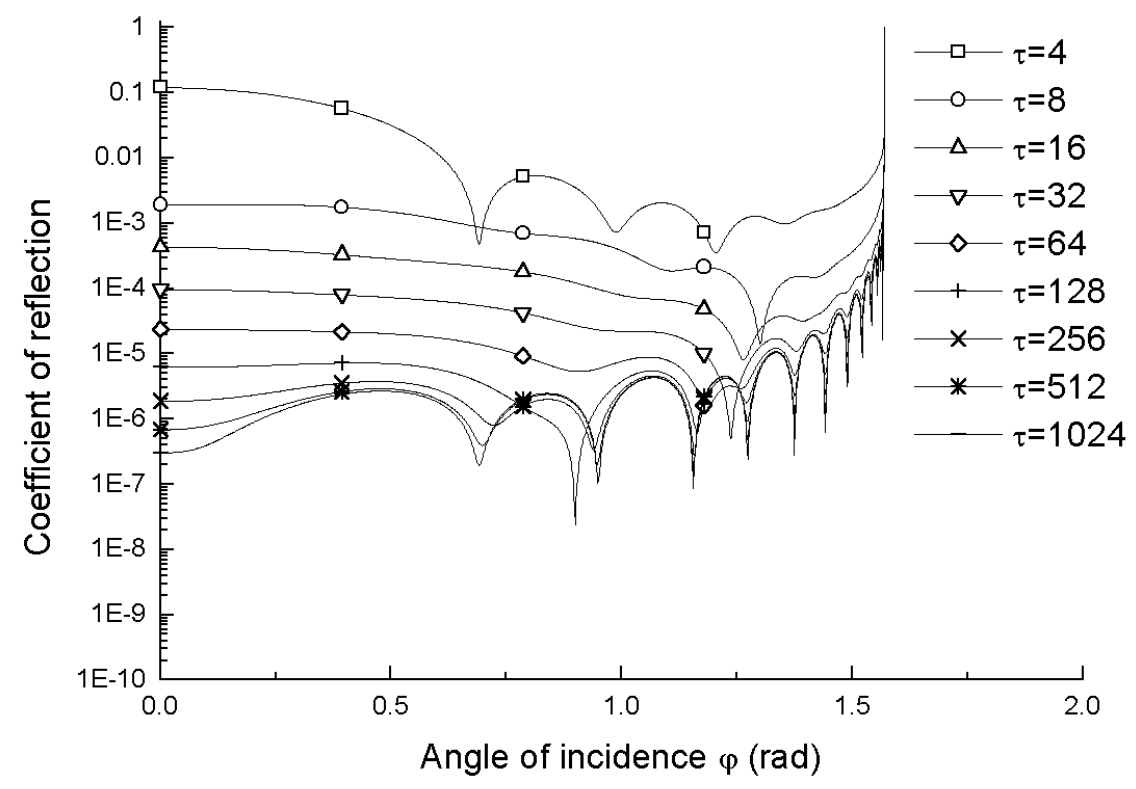

FIG. 14 Coefficient of reflection of the APML-SSA $(\mathrm{p}=1)$ as a function of the angle of incidence. 


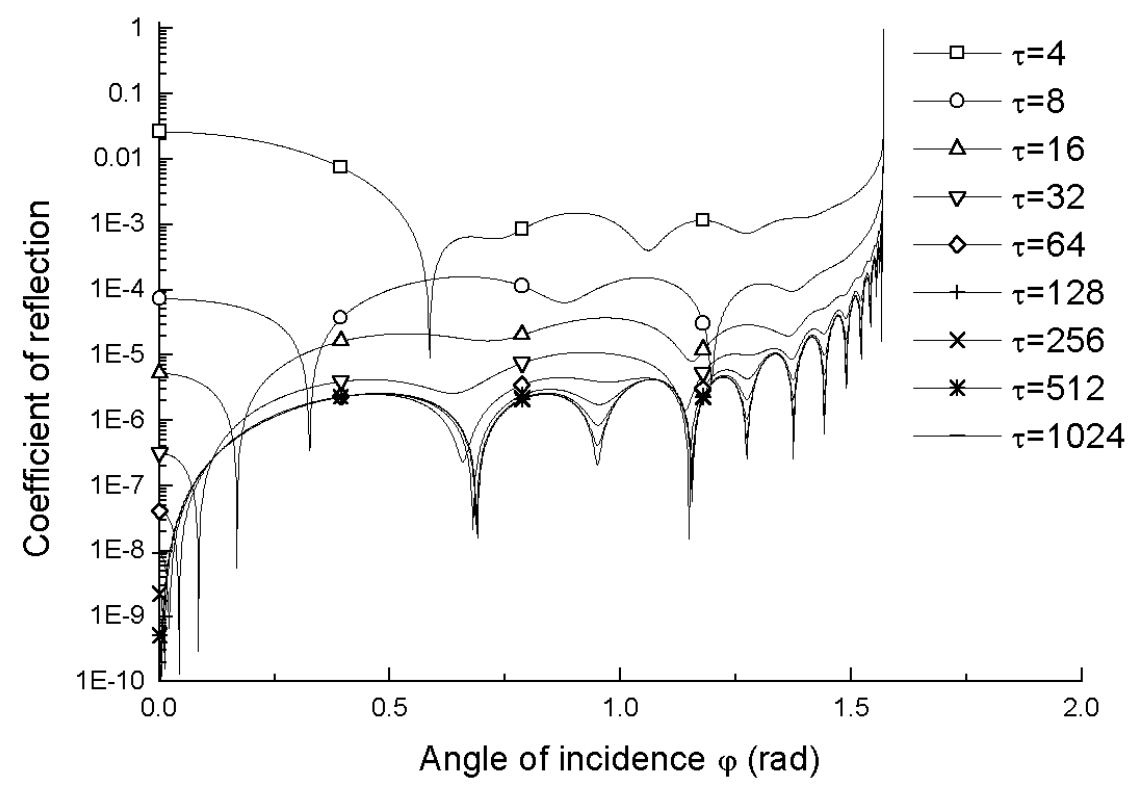

FIG. 15 Coefficient of reflection of the APML-LWA $(p=-1 \ldots+1)$ as a function of the angle of incidence. 


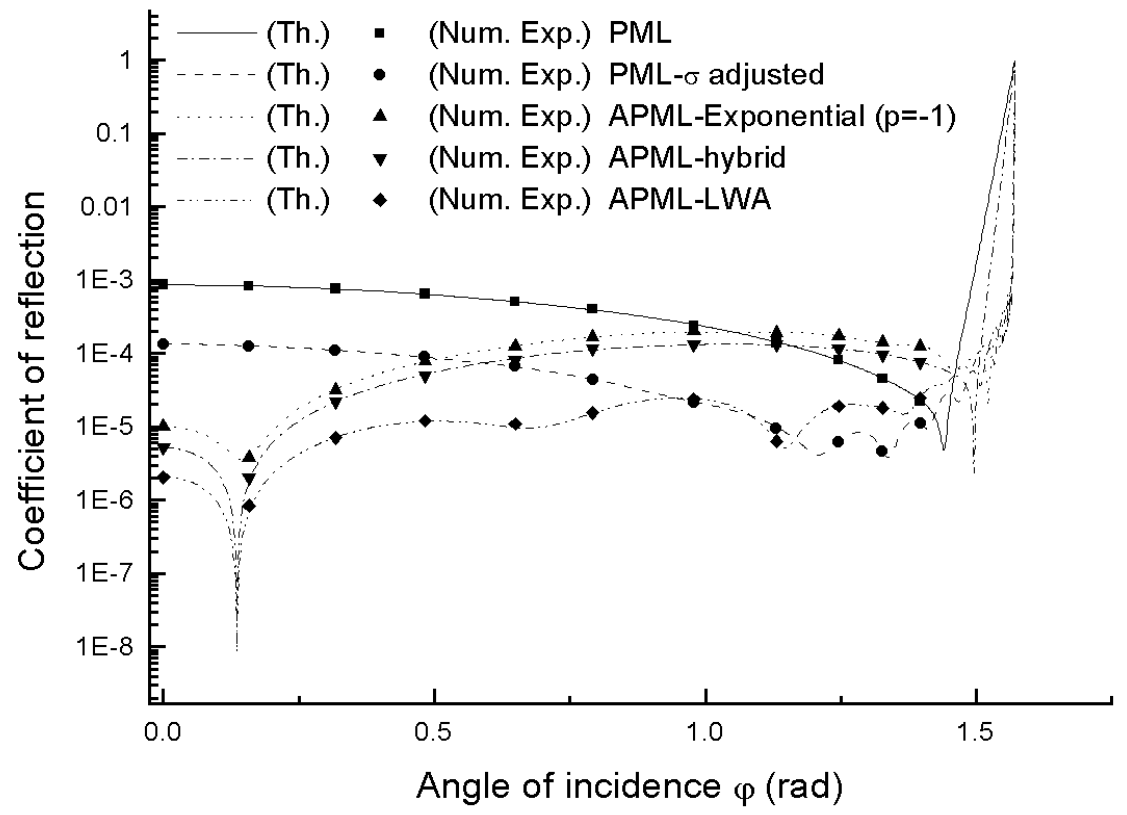

FIG. 16 Comparison coefficient of reflection of PML and APML as a function of the angle of incidence for $\tau=2 \pi / \omega \simeq 20 \delta x / c$. 